\title{
A WASSERSTEIN GRADIENT FLOW APPROACH TO POISSON-NERNST-PLANCK EQUATIONS
}

\author{
DAVID KINDERLEHRER, LÉONARD MONSAINGEON, AND XIANG XU
}

\begin{abstract}
The Poisson-Nernst-Planck system of equations used to model ionic transport is interpreted as a gradient flow for the Wasserstein distance and a free energy in the space of probability measures with finite second moment. A variational scheme is then set up and is the starting point of the construction of global weak solutions in a unified framework for the cases of both linear and nonlinear diffusion. The proof of the main results relies on the derivation of additional estimates based on the flow interchange technique developed by Matthes, McCann, and Savaré in 25.
\end{abstract}

\section{CONTEnTs}

1. Introduction

2. Formal Wasserstein gradient flow

3. Study of the energy functionals

4. Minimizing scheme and discrete estimates

5. Convergence to a weak solution

6. Appendix

References

\section{INTRODUCTION}

The Poisson-Nernst-Planck (PNP) system of equations [18, 24] is the principal description of ionic transport of several interacting species. It has been applied in a number of contexts ranging from electrical storage devices to molecular biology, at times coupled to Navier-Stokes or other systems. The basic system is to find $u(t, x) \geq 0$ and $v(t, x) \geq 0$ satisfying

$$
\begin{aligned}
\partial_{t} u & =\Delta u^{m}+\operatorname{div}(u \nabla(U+\psi)), \\
\partial_{t} v & =\Delta v^{m}+\operatorname{div}(v \nabla(V-\psi)), \quad t \geq 0, x \in \mathbb{R}^{d}, d \geq 3, \\
-\Delta \psi & =u-v,
\end{aligned}
$$

for some suitable initial conditions $\left.u\right|_{t=0}=u^{0}$ and $\left.v\right|_{t=0}=v^{0}$. The unknowns $u$ and $v$ represent the density of some positively and negatively charged particles. Here $m \geq 1$ is a chosen fixed nonlinear diffusion exponent. Note that (1.1) formally preserves the $L^{1}$ mass

$$
\int_{\mathbb{R}^{d}} u(t, x) \mathrm{d} x=\int_{\mathbb{R}^{d}} u^{0}(x) \mathrm{d} x \quad \text { and } \quad \int_{\mathbb{R}^{d}} v(t, x) \mathrm{d} x=\int_{\mathbb{R}^{d}} v^{0}(x) \mathrm{d} x
$$

for all $t \geq 0$, which physically represents the conservation of total charge of the individual species. For initial masses $\int_{\mathbb{R}^{d}} u^{0}(x) \mathrm{d} x=\int_{\mathbb{R}^{d}} v^{0}(x) \mathrm{d} x$, a simple rescaling of time and space allows normalization of masses to unity, and without loss of generality 
we consider $u(t, x), v(t, x)$ to be probability densities. The external potentials $U(x)$ and $V(x)$ are prescribed and sufficiently smooth. $\psi(x)$ from the Gauss Law is the self-consistent electrostatic potential created by the two charge carriers according to the last equation in (1.1). The first two equations in (1.1) are called Nernst-Planck equations and describe electro-diffusion and electrophoresis according to the Fick and Kohlrausch laws, respectively, while the last equation in (1.1) corresponds to the electrostatic Poisson law. The boundary condition for this coupling equation will always be understood in the sense of the Newtonian potential: we shall always implicitly write

$$
-\Delta \psi=u-v \quad \Leftrightarrow \quad \psi=G *(u-v)=(-\Delta)^{-1}(u-v),
$$

where

$$
G(x):=\frac{1}{d(d-2) \omega_{d}}|x|^{2-d}
$$

is the fundamental solution of $-\Delta$ in $\mathbb{R}^{d}$ and $\omega_{d}$ the volume of the unit ball in $\mathbb{R}^{d}$ (for $d \geq 3$ ).

In some PNP models, an extra background doping profile $C(x)$ is considered resulting in the modified potential $-\Delta \psi^{\prime}=u-v+C$. With suitable assumptions on $C(x)$ this can be easily eliminated replacing the external potentials $U$ by $U+\psi_{C}$ and $V$ by $V-\psi_{C}$, where $\psi_{C}=(-\Delta)^{-1} C$.

There is a vast literature on well-posedness and long time behavior of the system (1.1). We refer to [6, 13, 14, 17] and references therein for bounded domains, and [3, 4, 17, 12, 21] for the whole space problem. Different from these papers, our contribution is to show that the system of equations (1.1), governing drift, diffusion and reaction of charged species, possesses a gradient flow structure as viewed on the metric space of probability measures on $\mathbb{R}^{d}$ endowed with the quadratic Wasserstein distance, in essence the weak-* topology (see also [28, 31, 36] for various discussions). Therefore, variational methods may be introduced to prove global existence of weak solutions (see definition below). Motivation for this in terms of energy dissipation is offered below. Also, we provide a unified framework both for linear $m=1$ and nonlinear $m>1$ diffusions. To the best of our knowledge well-posedness in the whole space for $m>1$ usually requires either high integrability of the initial data or initial gradient regularity. We would like to stress that we need here no such hypotheses and that our result merely requires some low initial integrability, defined in terms of the diffusion exponent $m \geq 1$ and dimension $d \geq 3$ only (which we think is sharp, see discussion after the proof of Proposition 4.4 page 19). Also, we do not need compatibility conditions between $m \geq 1$ and $d \geq 3$. This is in contrast with the Patlak-Keller-Segel chemotaxis models [8, 9, for which critical mass phenomena may occur depending on whether $m$ is larger or smaller than the scale-invariant exponent $m_{*}(d)=2-\frac{2}{d}$, see e.g. [10] for the critical parabolic-parabolic case. This is mainly due to the fact that the self-induced drifts are repulsive here, while they are self-attractive in the Keller-Segel models, thus leading to aggregation and blow-up in finite time.

It was shown in [16, 30] that certain scalar diffusion equations can be interpreted as gradient flows in metric spaces and the literature concerning this issue is steadily growing (see [2] and references therein). It is thus a question of great interest to apply such ideas to study systems of equations. In contrast, there are only a few examples for systems. For related studies, we refer to [8, 9, 10, 15, 20, 22, 26, 37], where the energy functional is involved with the Wasserstein metric and existence 
theorems using a minimizing movement scheme for corresponding evolution problems 3 are presented.

In [16], the linear Fokker-Planck equation

$$
\partial_{t} \rho=\sigma \Delta \rho+\operatorname{div}(\rho \nabla \varphi)
$$

is regarded as the gradient flow of a free energy consisting of the Boltzmann entropy with a potential $\varphi$,

$$
\mathcal{F}(\rho)=\int_{\mathbb{R}^{d}}(\varphi \rho+\sigma \rho \log \rho) \mathrm{d} x,
$$

with respect to the quadratic Wasserstein metric. There may be many Lyapunov functions associated to a differential equation. The result of [16 means that dissipation for the free energy $\mathcal{F}$ determines the Fokker-Planck Equation. The same idea was later employed in [30] and, in addition, to derive long-time asymptotics for the Porous Medium Equation (PME). Since the system (1.1) can be viewed as two Fokker-Planck equations (when $m=1$ ) or Porous Medium equations (when $m>1$ ) in $u, v$ coupled by means of a Poisson kernel, we are motivated to extend these ideas to study our coupled system. Inspired by [30] and [35], we shall in fact discover that the PNP system can be seen as a gradient flow driven by the free energy

$$
\begin{array}{ll}
\mathcal{E}(u, v):=\int_{\mathbb{R}^{d}}\left(u \log u+v \log v+u U+v V+\frac{|\nabla \psi|^{2}}{2}\right) \mathrm{d} x \quad \text { if } m=1, \\
\mathcal{E}(u, v):=\int_{\mathbb{R}^{d}}\left(\frac{u^{m}}{m-1}+\frac{v^{m}}{m-1}+u U+v V+\frac{|\nabla \psi|^{2}}{2}\right) \mathrm{d} x \quad \text { if } m>1 .
\end{array}
$$

We further motivate this approach informally by discussing the relationship between the dissipation relation and the weak-* topology in terms of the WassersteinRubinstein-Kantorovich distance, or simply the Wasserstein distance. We follow [36] and consider for illustration the case $m=1$. Set

$$
\begin{aligned}
& \varphi(u, v)=u \log u+v \log v+u U+v V+\frac{1}{2}|\nabla \psi|^{2}, \text { so that } \\
& \mathcal{E}(u, v)=\int_{\mathbb{R}^{d}} \varphi(u, v) \mathrm{d} x .
\end{aligned}
$$

Given a process or an evolution $(u(t), v(t))$, during an interval $(T, T+h)$ the change in energy is

$$
\left.\mathcal{E}(u, v)\right|_{T+h}-\int_{T}^{T+h} \int_{\mathbb{R}^{d}} \frac{\mathrm{d}}{\mathrm{d} t} \varphi(u, v) \mathrm{d} x \mathrm{~d} t=\left.\mathcal{E}(u, v)\right|_{T}
$$

This, (1.4), is the dissipation equality or inequality and the density of the middle term

$$
\mathcal{D}=-\int_{\mathbb{R}^{d}} \frac{\mathrm{d}}{\mathrm{d} t} \varphi(u, v) \mathrm{d} x
$$

is the dissipation density along the trajectory. Writing

$$
\frac{\mathrm{d}}{\mathrm{d} t} \varphi(u, v)=\varphi_{u} \frac{\mathrm{d} u}{\mathrm{~d} t}+\varphi_{v} \frac{\mathrm{d} v}{\mathrm{~d} t},
$$

we must ascribe a meaning to

$$
\frac{\mathrm{d} u}{\mathrm{~d} t}, \frac{\mathrm{d} v}{\mathrm{~d} t}
$$

to render the system dissipative, that is, so that (1.5) is positive. To begin we calculate the terms in (1.6). Keeping in mind (1.2), one checks that

$$
\frac{\delta}{\delta u}\left(\frac{1}{2}|\nabla \psi|^{2}\right)=\psi \text { and } \frac{\delta}{\delta v}\left(\frac{1}{2}|\nabla \psi|^{2}\right)=-\psi,
$$


which leads to

$$
\varphi_{u}(u, v)=\log u+U+\psi+1 \text { and } \varphi_{v}=\log v+V-\psi+1 .
$$

Let us now employ the Poisson-Nernst-Planck equations (1.1). Substituting into (1.5) and integrating by parts gives

$$
\mathcal{D}=\int_{\mathbb{R}^{d}}\left\{\left|\frac{\nabla u}{u}+\nabla(U+\psi)\right|^{2} u+\left|\frac{\nabla v}{v}+\nabla(V-\psi)\right|^{2} v\right\} \mathrm{d} x
$$

Introduce

$$
\begin{aligned}
& w=-\left(\frac{\nabla u}{u}+\nabla(U+\psi)\right) \text { and } \omega=-\left(\frac{\nabla v}{v}+\nabla(V-\psi)\right) \text { so that } \\
& u_{t}+\operatorname{div}(w u)=0 \text { and } v_{t}+\operatorname{div}(\omega v)=0 .
\end{aligned}
$$

We have that

$$
\int_{T}^{T+h} \mathcal{D} \mathrm{d} t=\int_{T}^{T+h} \int_{\mathbb{R}^{d}}|w|^{2} u \mathrm{~d} x \mathrm{~d} t+\int_{T}^{T+h} \int_{\mathbb{R}^{d}}|\omega|^{2} v \mathrm{~d} x \mathrm{~d} t
$$

where the pairs $(u, w),(v, \omega)$ satisfy the continuity equations (1.7). This represents a trial in $d_{W}$, the quadratic Wasserstein metric, using the Benamou-Brenier formulation [5], where

$$
\begin{aligned}
& \frac{1}{h} d_{W}\left(\left.u\right|_{T+h},\left.u\right|_{T}\right)^{2}=\inf \int_{T}^{T+h} \int_{\mathbb{R}^{d}}|w|^{2} u \mathrm{~d} x \mathrm{~d} t \text { and } \\
& \frac{1}{h} d_{W}\left(\left.v\right|_{T+h},\left.v\right|_{T}\right)^{2}=\inf \int_{T}^{T+h} \int_{\mathbb{R}^{d}}|\omega|^{2} v \mathrm{~d} x \mathrm{~d} t
\end{aligned}
$$

taken over all pairs $(u, w)$ and $(v, \omega)$ satisfying the continuity equations

$u_{t}+\operatorname{div}(w u)=0$ and $v_{t}+\operatorname{div}(\omega v)=0$, and

the initial and terminal conditions.

The calculation shows that the dissipation relation (1.7) for $\mathcal{E}$ and the PNP system are closely related to the Wasserstein distance. We could write, in fact,

$$
\frac{1}{h} d_{W}\left(\left.u\right|_{T+h},\left.u\right|_{T}\right)^{2}+\frac{1}{h} d_{W}\left(\left.v\right|_{T+h},\left.v\right|_{T}\right)^{2}+\left.\mathcal{E}(u, v)\right|_{T+h} \leq\left.\mathcal{E}(u, v)\right|_{T}
$$

suggesting an implicit scheme which leads to a gradient flow. This is nearly correct. As is well known, a factor of $1 / 2$ must be inserted; see below (1.8).

We now turn to the precise formulation. Denoting $\mathcal{P}\left(\mathbb{R}^{d}\right)$ the set of Borel probability measures on $\mathbb{R}^{d}$ with finite second moments and $d_{W}$ the quadratic Wasserstein distance as before, the underlying space will be here $(u, v) \in \mathcal{P}\left(\mathbb{R}^{d}\right) \times \mathcal{P}\left(\mathbb{R}^{d}\right)$ and will inherit a natural differential structure from that of $\left(\mathcal{P}\left(\mathbb{R}^{d}\right), d_{W}\right)$ - see section 2 for details. The total free energy (1.3) is a combination of the well-known internal (diffusive entropy) and potential energies for each species, and, although unclear at this stage, the coupling Dirichlet energy $\frac{1}{2} \int_{\mathbb{R}^{d}}|\nabla \psi|^{2} \mathrm{~d} x$ falls into the category of so-called interaction energies. See [35] for an introduction.

Following [16, we shall construct weak solutions $z=(u, v)$ as follows. Given suitable initial data $z^{0}=\left(u^{0}, v^{0}\right)$ and some small time step $h \in(0,1)$ we first construct a discrete sequence $\left\{z_{h}^{(n)}\right\}_{n \in \mathbb{N}}$ solution to the Jordan-Kinderlehrer-Otto or JKO implicit scheme

$$
\begin{aligned}
& z_{h}^{(0)}=z^{0}, \\
& z_{h}^{(n+1)} \in \underset{\mathcal{K} \times \mathcal{K}}{\operatorname{Argmin}}\left\{\frac{1}{2 h} d^{2}\left(\cdot, z_{h}^{(n)}\right)+\mathcal{E}(\cdot)\right\}
\end{aligned}
$$


Here $\mathcal{E}(z)=\mathcal{E}(u, v)$ is the total free energy (1.3), $d^{2}$ is the (squared) distance on the product space inherited from $d_{W}$, and $\mathcal{K} \subset \mathcal{P}$ the set of admissible minimizers defined later on. As is classical by now, one obtains interpolating solutions $\left\{z_{h}(t)\right\}_{h}=\left\{u_{h}(t), z_{h}(t)\right\}_{h}$ defined for all $t \geq 0$, piecewise constant in time, and satisfying a coupled system of two Euler-Lagrange equations. We shall then prove that as $h \rightarrow 0$ one recovers a weak solution $(u(t), v(t))=z(t)=\lim _{h \rightarrow 0} z_{h}(t)$ of (1.1). There are several challenges in this program.

In handling the $\int_{\mathbb{R}^{d}}|\nabla \psi|^{2} \mathrm{~d} x$ coupling term, some intrinsic difficulties arise due both to the specific Poisson kernel and to the nonscalar setting. First, as neither the external potentials nor $G$ are convex the free energy $\mathcal{E}$ is not displacement convex in the sense of McCann [27] and we cannot simply apply the standard procedures as in [2]. Secondly, due to the singular nature of the kernel $G(x)=C /|x|^{d-2}$ both the existence of minimizers in (1.8) and derivation of the corresponding Euler-Lagrange equations become delicate, see in particular the discussions in Proposition 3.1 and Proposition 4.4 for details. In order to tackle this issue we used the "flow interchange" technique that originates in [25] and was later used in [10, 22] to obtain some integrability improvement and gradient regularity of the minimizers, see Proposition 3.4 and Proposition 3.5 below. The highlight of the argument is the propagation of initial $L^{p}\left(\mathbb{R}^{d}\right)$ regularity established in Proposition 3.4. In addition to being technically essential here, this propagation of initial regularity allowed us to obtain a natural $L^{\infty}\left(\mathbb{R}^{d}\right.$ ) estimate (see Theorems 2 2-3), which to the best of our knowledge was unknown for the PNP system in the whole space. We also believe that the very same argument could be employed for similar problems in order to show propagation of initial regularity, which is usually a delicate point in the mass transport framework.

The rest of the paper is organized as follows. In Section 2 we recall well-known facts in optimal transport theory and briefly describe the differential structure of the product space. We then formally derive the Wasserstein gradient flow structure of the system (1.1) and state the main existence results. In Section 3 we study the relevant energy functionals, and establish improved regularity of their minimizers. In Section 4 we fix a time step $h>0$ small enough and consider the minimizing scheme. We obtain approximate discrete solutions $\left\{u_{h}, v_{h}\right\}_{h}$ and derive the corresponding Euler-Lagrange equations. In Section 5 we take the limit $h \rightarrow 0$ and show that the convergence $(u, v)=\lim _{h \rightarrow 0}\left(u_{h}, v_{h}\right)$ is strong enough to retrieve a weak solution. This last section also contains the proof of the main theorems.

Notation Convention. Unless otherwise specified, $\langle\cdot, \cdot\rangle$ and $\cdot$ denote inner product of elements in $\mathbb{R}^{d}, \mathcal{P}$ denotes $\mathcal{P}\left(\mathbb{R}^{d}\right)$, and $\mathcal{P}^{a c}$ denotes $\mathcal{P}^{a c}\left(\mathbb{R}^{d}\right)$. If clear from the context we shall often omit the subscripts $m=1$ or $m>1$. If $1 \leq p \leq \infty$, we denote by $p^{\prime}=\frac{p}{p-1}$ the conjugate Lebesgue exponent.

\section{Formal Wasserstein Gradient Flow}

From now on we assume that the external potentials are quadratic at infinity, i-e

$$
\begin{gathered}
C_{1}|x|^{2} \leq U(x), V(x) \leq C_{2}\left(1+|x|^{2}\right) \\
|\nabla U(x)|,|\nabla V(x)| \leq C_{3}(1+|x|) \\
\|\Delta U\|_{L^{\infty}\left(\mathbb{R}^{d}\right)},\|\Delta V\|_{L^{\infty}\left(\mathbb{R}^{d}\right)} \leq C_{4},
\end{gathered}
$$

for some generic positive constants $C_{i}$. Note that $U, V$ need not be uniformly convex as is often assumed, so that we allow here multiple wells. Although these assumptions 
on the potentials could be weakened we assume here strict confinement $C_{1}>0$ for the ease of exposition, and we do not seek optimal generality.

We also introduce the admissible set

$$
\mathcal{K}:= \begin{cases}\mathcal{K}_{1}:=\mathcal{P}^{a c}\left(\mathbb{R}^{d}\right) \cap L^{1} \log L^{1}\left(\mathbb{R}^{d}\right) & \text { if } m=1 \\ \mathcal{K}_{m}:=\mathcal{P}^{a c}\left(\mathbb{R}^{d}\right) \cap L^{m}\left(\mathbb{R}^{d}\right) & \text { if } m>1\end{cases}
$$

and for reasons that shall become clear later on we shall always consider initial data

$$
u^{0}, v^{0} \in \mathcal{K} \cap L^{r_{0}}\left(\mathbb{R}^{d}\right) \quad \text { for some } r_{0}>\max \{m, 2 d /(d+1)\} .
$$

Essentially $u^{0}, v^{0} \in L^{m} \cap L^{2 d /(d+2)}\left(\mathbb{R}^{d}\right)$ ensures that the initial energy $\left(u^{0}, v^{0}\right)$ is finite, while $L^{2 d /(d+1)}\left(\mathbb{R}^{d}\right)$ regularity will ensure that the self-induced drifts $u \nabla \Psi, v \nabla \Psi \in$ $L^{1}\left(\mathbb{R}^{d}\right)$ for all times. We believe that $r=\max \{m, 2 d /(d+1)\}$ should be admissible, but for technical compactness issues we have to assume here slightly better $L^{r_{0}}\left(\mathbb{R}^{d}\right)$ integrability for some $r_{0}>r$ arbitrarily close. See the proof of Theorem 4 later on for details.

For $\rho, u, v \geq 0$ let us define the usual Boltzmann entropy

$$
\mathcal{H}(\rho):=\int_{\mathbb{R}^{d}} \rho \log \rho \mathrm{d} x
$$

the diffusion energy

$$
\begin{aligned}
& \mathcal{E}_{\text {diff }}(u, v):=\int_{\mathbb{R}^{d}}(u \log u+v \log v) \mathrm{d} x \text { if } m=1 \text { and } \\
& \mathcal{E}_{\text {diff }}(u, v):=\frac{1}{m-1} \int_{\mathbb{R}^{d}}\left(u^{m}+v^{m}\right) \mathrm{d} x \text { if } m>1
\end{aligned}
$$

and the external potential energy

$$
\mathcal{E}_{\text {ext }}(u, v):=\int_{\mathbb{R}^{d}}(u U+v V) \mathrm{d} x .
$$

Note that, with our assumptions, $\mathcal{E}_{\text {diff }}, \mathcal{E}_{\text {ext }}$ are finite for all $(u, v) \in \mathcal{K} \times \mathcal{K}$. For $\psi=(-\Delta)^{-1}(u-v)=G *(u-v)$ we define now the coupling energy

$$
\mathcal{E}_{\mathrm{cpl}}(u, v):=\frac{1}{2} \int_{\mathbb{R}^{d}}|\nabla \psi|^{2} \mathrm{~d} x
$$

which is the energy of the self-induced electric potential. Note that, at least formally,

$$
\begin{aligned}
\int_{\mathbb{R}^{d}}|\nabla \psi|^{2} \mathrm{~d} x & =\int_{\mathbb{R}^{d}}(-\Delta \psi) \psi \mathrm{d} x=\int_{\mathbb{R}^{d}}(u-v) G *(u-v) \mathrm{d} x \\
& =\iint_{\mathbb{R}^{d} \times \mathbb{R}^{d}}[u-v](x) G(x-y)[u-v](y) \mathrm{d} x \mathrm{~d} y
\end{aligned}
$$

falls into the category of interaction energies

$$
\iint_{\mathbb{R}^{d} \times \mathbb{R}^{d}} \rho(x) K(x, y) \rho(y) \mathrm{d} x \mathrm{~d} y
$$

treated in [35]. To sum up, the total free energy $\mathcal{E}=\mathcal{E}_{\text {diff }}+\mathcal{E}_{\text {ext }}+\mathcal{E}_{\text {cpl }}$ is given by (1.3)

For given probability measures $\mu, \nu \in \mathcal{P}\left(\mathbb{R}^{d}\right)$ we denote the squared (quadratic) Wasserstein distance by

$$
d_{W}^{2}(\mu, \nu)=\inf _{\gamma \in \Gamma(\mu, \nu)} \iint_{\mathbb{R}^{d} \times \mathbb{R}^{d}}|x-y|^{2} \mathrm{~d} \gamma(x, y),
$$

where $\Gamma(\mu, \nu) \subset \mathcal{P}\left(\mathbb{R}_{x}^{d} \times \mathbb{R}_{y}^{d}\right)$ is the set of admissible joint distributions with $x$ and $y$ marginals $\mu, \nu$ respectively. We recall from [35] that $\left(\mathcal{P}, d_{W}\right)$ is a metric space and 
that $d_{W}$ metrizes the weak convergence of measures. When $\mu \in \mathcal{P}_{2}^{a c}$ is moreover absolutely continuous with respect to the Lebesgue measure $\mathrm{d} \mu(x) \ll \mathrm{d} x$, the square Wasserstein distance can also be computed by the Benamou-Brenier theorem [5] as already noted.

Theorem 1 (Existence of optimal maps, 35]). Let $\mu \in \mathcal{P}_{2}^{a c}\left(\mathbb{R}^{d}\right)$ and $\nu \in \mathcal{P}_{2}^{a c}\left(\mathbb{R}^{d}\right)$. There exists a unique optimal transport map $T=\nabla \varphi \in L^{2}\left(\mathbb{R}^{d} ; \mathrm{d} \mu\right)$ for some convex function $\varphi$ such that

$$
\nu=T_{\#} \mu: \quad \forall f \in \mathcal{C}_{c}\left(\mathbb{R}^{d}\right), \int_{\mathbb{R}^{d}} f(y) \mathrm{d} \nu(y)=\int_{\mathbb{R}^{d}} f \circ T(x) \mathrm{d} \mu(x)
$$

and

$$
d_{W}^{2}(\mu, \nu)=\int_{\mathbb{R}^{d}}|x-T(x)|^{2} \mathrm{~d} \mu(x)
$$

Our interest here is a system so we endow $\mathcal{P}\left(\mathbb{R}^{d}\right) \times \mathcal{P}\left(\mathbb{R}^{d}\right)$ with the natural product distance

$$
d^{2}\left(z, z^{\prime}\right)=d_{W}^{2}\left(u, u^{\prime}\right)+d_{W}^{2}\left(v, v^{\prime}\right)
$$

for all $z=(u, v)$ and $z^{\prime}=\left(u^{\prime}, v^{\prime}\right)$ in $\mathcal{P} \times \mathcal{P}$. It is well known [30, 35] that $\left(\mathcal{P}, d_{W}\right)$ enjoys a natural differential structure defined by means of continuity equations, so that our product space also has the same differential structure. This permits us to differentiate real-valued functions $\mathcal{F}$ on the product space, and defines the corresponding Wasserstein gradient by the chain rule $\frac{\mathrm{d}}{\mathrm{d} t} \mathcal{F}\left(z_{t}\right)=\operatorname{grad}_{W} \mathcal{F}\left(z_{t}\right) \cdot \frac{\mathrm{d} z_{t}}{\mathrm{~d} t}$. We show now that (1.1) is really the gradient flow

$$
\frac{\mathrm{d} z}{\mathrm{~d} t}=-\operatorname{grad}_{W} \mathcal{E}(z), \quad z(t)=\left(\begin{array}{c}
u(t) \\
v(t)
\end{array}\right) .
$$

In terms of ordinary calculus of variations, we recall that this is achieved by variation of domain or, in fluid dynamical terms, by Lagrangian variations, [16]. To this end, let us split for convenience the coupling Poisson equation $-\Delta \psi=u-v$ as

$$
\psi=\psi_{u}-\psi_{v} \quad \text { with }\left\{\begin{array}{l}
-\Delta \psi_{u}=u \Leftrightarrow \psi_{u}=G * u, \\
-\Delta \psi_{v}=v \Leftrightarrow \psi_{v}=G * v .
\end{array}\right.
$$

Formally differentiating the diffusive energy (2.4) with respect to $u$ (resp. $v$ ), a by now classical computation [30, 35] leads to the $\Delta u^{m}$ (resp. $\left.\Delta v^{m}\right)$ term in (1.1). Similarly, differentiating the external energy (2.5) with respect to $u$ and $v$ classically gives rise to $\nabla \cdot(u \nabla U)$ and $\nabla \cdot(v \nabla V)$ in (1.1). In order to differentiate the coupling term we first use the formal integration by parts (2.7) and then exploit the symmetry $G(x-y)=G(y-x)$ to expand

$$
\begin{aligned}
\mathcal{E}_{\mathrm{cpl}}(u, v) & =\frac{1}{2} \iint_{\mathbb{R}^{d} \times \mathbb{R}^{d}}[u-v](x) G(x-y)[u-v](y) \mathrm{d} x \mathrm{~d} y \\
& =\frac{1}{2} \int_{\mathbb{R}^{d}}[u(G * u)+v(G * v)] \mathrm{d} x-\iint_{\mathbb{R}^{d} \times \mathbb{R}^{d}} u(x) G(x-y) v(y) \mathrm{d} x \mathrm{~d} y .
\end{aligned}
$$

Differentiating with respect to $u$, it is well known 35, that the first integral gives the corresponding $\nabla \cdot(u \nabla(G * u))+0=\nabla \cdot\left(u \nabla \psi_{u}\right)$ term. Rewriting the remaining cross term

$$
-\iint_{\mathbb{R}^{d} \times \mathbb{R}^{d}} u G v \mathrm{~d} x=-\int_{\mathbb{R}^{d}} u(G * v) \mathrm{d} x=-\int_{\mathbb{R}^{d}} u \psi_{v} \mathrm{~d} x,
$$

and noting that $\psi_{v}$ is independent of $u$, it is again well known that this term gives rise to $-\nabla \cdot\left(u \nabla \psi_{v}\right)$. Summing up we obtain $\nabla \cdot\left(u \nabla\left(\psi_{u}-\psi_{v}\right)\right)=\nabla \cdot(u \nabla \psi)$ as in 
the first equation of (1.1). Similarly differentiating with respect to $v$ we obtain the $-\nabla \cdot(v \nabla \psi)$ term appearing in the second component.

Though very general notions of solutions related to Energy Dissipation Equality (EDE) or Evolution Variational Inequality (EVI) can be used for abstract gradient flows in metric spaces, [1, 2, we use the more direct framework, introducing some features later for the implementation of the flow-interchange method.

Definition 2.1. A pair $u, v:(0, \infty) \times \mathbb{R}^{d} \rightarrow \mathbb{R}^{+}$is a global weak solution if $u, v \in$ $\mathcal{C}([0, \infty) ; \mathcal{P}), u(t), v(t) \rightarrow u^{0}, v^{0}$ in $\left(\mathcal{P}, d_{W}\right)$ as $t \searrow 0, \nabla u^{m}, \nabla v^{m}, u \nabla U, v \nabla V, u \nabla \psi$ and $v \nabla \psi \in L^{2}\left(0, T ; L^{1}\left(\mathbb{R}^{d}\right)\right)$ for all $T>0$, and for any fixed $\varphi \in \mathcal{C}_{c}^{\infty}\left(\mathbb{R}^{d}\right)$

$$
\begin{aligned}
\frac{\mathrm{d}}{\mathrm{d} t} \int_{\mathbb{R}^{d}} u(t, x) \varphi \mathrm{d} x= & -\int_{\mathbb{R}^{d}}\left\langle\nabla u^{m}(t, x), \nabla \varphi\right\rangle \mathrm{d} x-\int_{\mathbb{R}^{d}} u(t, x)\langle\nabla U, \nabla \varphi\rangle \mathrm{d} x \\
& -\int_{\mathbb{R}^{d}} u(t, x)\langle\nabla \psi(t, x), \nabla \varphi\rangle \mathrm{d} x,
\end{aligned}
$$

and

$$
\begin{aligned}
\frac{\mathrm{d}}{\mathrm{d} t} \int_{\mathbb{R}^{d}} v(t, x) \varphi \mathrm{d} x= & -\int_{\mathbb{R}^{d}}\left\langle\nabla v^{m}(t, x), \nabla \varphi\right\rangle \mathrm{d} x-\int_{\mathbb{R}^{d}} v(t, x)\langle\nabla V, \nabla \varphi\rangle \mathrm{d} x \\
& +\int_{\mathbb{R}^{d}} v(t, x)\langle\nabla \psi(t, x), \nabla \varphi\rangle \mathrm{d} x
\end{aligned}
$$

hold in the sense of distributions $\mathcal{D}^{\prime}(0, \infty)$ with $\psi(t, x)=G *[u-v](t, x)$ a.e. $(t, x) \in$ $(0, \infty) \times \mathbb{R}^{d}$.

We observe that $L_{l o c}^{2}\left([0, \infty) ; L^{1}\left(\mathbb{R}^{d}\right)\right)$ could be replaced by $L_{l o c}^{1}\left((0, \infty) \times \mathbb{R}^{d}\right)$ in the above definition, which is enough for all the integrals in (2.8)-(2.9) to make sense. In any case the weak solutions constructed here would still enjoy strong regularity in the end, and our choice of including the regularity in the definition of weak solutions is purely practical.

In this setting our main result is

Theorem 2 (Existence of solutions for $m>1$ ). Fix $m>1$ and initial data $u^{0}, v^{0}$ as in (2.3). Then there exists a global weak solution $(u, v)$ with

$$
\begin{aligned}
& u, v \in L^{\infty}\left(0, \infty ; L^{m}\left(\mathbb{R}^{d}\right) \cap L^{1}\left(\mathbb{R}^{d},\left(1+|x|^{2}\right) \mathrm{d} x\right)\right) \\
& \nabla \psi \in L^{\infty}\left(0, \infty ; L^{2}\left(\mathbb{R}^{d}\right)\right) \\
& u^{\frac{m}{2}}, v^{\frac{m}{2}} \in L^{2}\left(0, T ; H^{1}\left(\mathbb{R}^{d}\right)\right) \\
& u, v \in L^{\infty}\left(0, T ; L^{p}\left(\mathbb{R}^{d}\right)\right), \quad \forall p \in[1,2 d /(d+1)],
\end{aligned}
$$

for all $T>0$, and

$$
\mathcal{E}(u(t), v(t)) \leq \mathcal{E}\left(u^{0}, v^{0}\right) \quad \text { for a.e. } t \geq 0 .
$$

If we further assume that $u^{0}, v^{0} \in L^{p}\left(\mathbb{R}^{d}\right)$ for some $p \in[1, \infty]$, then $\forall \tau \geq 0$,

$$
\sup _{t \in[0, \tau]}\left(\|u(t)\|_{L^{p}\left(\mathbb{R}^{d}\right)}+\|v(t)\|_{L^{p}\left(\mathbb{R}^{d}\right)}\right) \leq C e^{\lambda \tau}\left(\left\|u^{0}\right\|_{L^{p}\left(\mathbb{R}^{d}\right)}+\left\|v^{0}\right\|_{L^{p}\left(\mathbb{R}^{d}\right)}\right),
$$

with

$$
\lambda=\max \left\{\|\Delta U\|_{L^{\infty}\left(\mathbb{R}^{d}\right)},\|\Delta V\|_{L^{\infty}\left(\mathbb{R}^{d}\right)}\right\} .
$$

In the case of linear diffusion we have similarly 
Theorem 3 (Existence of solutions for $m=1$ ). The conclusions of Theorem 2 hold for $m=1$ if we replace $u, v \in L^{\infty}\left(0, \infty ; L^{m}\left(\mathbb{R}^{d}\right)\right)$ in $(2.10)$ by

$u, v \in L^{\infty}\left(0, \infty ; L^{1} \log L^{1}\left(\mathbb{R}^{d}\right)\right)$.

We would like to stress again that estimate (2.12) holds for $p=\infty$, and was not known in the whole space as far as we can tell. Since we interpret (1.1) as a gradient flow one could expect energy monotonicity $\mathcal{E}(t) \downarrow$. This would immediately follow from (2.11) and uniqueness of solutions. Unfortunately due to the lack of regularity and displacement convexity we were not able to prove uniqueness within the above class of weak solutions, and therefore we only retrieve an energy upper bound.

It is worth mentioning that the gradient flow structure of the PNP system and the above theorems are also valid in the bounded domain case, with some mild assumptions on the boundary and minor modifications of the proofs. Suppose $\Omega \subset$ $\mathbb{R}^{d}$ is a smooth bounded and convex domain, and consider the physically relevant boundary condition, that is, the no flux boundary condition

$$
\frac{\partial u}{\partial \nu}=\frac{\partial v}{\partial \nu}=\frac{\partial \psi}{\partial \nu}=0 \quad \text { on } \partial \Omega, \quad \int_{\partial \Omega} \psi \mathrm{d} x=0
$$

where $\nu$ is the unit outward normal on $\partial \Omega$. We also assume the external potentials satisfy

$$
\frac{\partial U}{\partial \nu}=\frac{\partial V}{\partial \nu}=0 \quad \text { on } \partial \Omega
$$

By [19] we know that the electrostatic potential can be represented as

$$
\psi(x)=\int_{\Omega} N(x, y)(u-v)(y) \mathrm{d} y, \quad \forall x \in \Omega .
$$

Here the (singular) kernel $N(x, y)=N(y, x)$ serves as a counterpart of the Green's function $G(x-y)$ in $\mathbb{R}^{d}$ for the Newton potential. Then we may argue in a similar way that the PNP system formally possesses the gradient flow structure, and the existence theorems can be proved in a similar but somewhat technically easier manner.

\section{StUdy OF THE ENERGY FUNCTIONALS}

In this section we study various properties of the two relevant energy functionals, namely the total free energy $\mathcal{E}$ and the functional (3.3) used in the JKO minimizing scheme. As already mentioned in the introduction, we use the flow interchange technique to establish some improved regularity for the minimizers, which will turn out to be crucial in the next sections.

For further use we recall here a particular case of the celebrated Hardy-LittlewoodSobolev (HLS) inequality:

Lemma 3.1 (Hardy-Littlewood-Sobolev, [23, 33]). In dimension $d \geq 3$ let $w \in$ $L^{p}\left(\mathbb{R}^{d}\right)$ and $\Phi=G * w$. Then

(1) If $1<p<d / 2$ there is a $C=C(p, d)$ such that

$$
\|\Phi\|_{L^{\frac{d p}{d-2 p}}\left(\mathbb{R}^{d}\right)} \leq C\|w\|_{L^{p}\left(\mathbb{R}^{d}\right)},
$$

while if $p=1$ there is a $C=C(d)$ such that

$$
\|\Phi\|_{L_{w}^{\frac{d}{d-2}\left(\mathbb{R}^{d}\right)}} \leq C\|w\|_{L^{1}\left(\mathbb{R}^{d}\right)} .
$$

(2) If $1<p<d$ there is a $C=C_{p, d}$ such that

$$
\|\nabla \Phi\|_{L^{\frac{d p}{d-p}}\left(\mathbb{R}^{d}\right)} \leq C\|w\|_{L^{p}\left(\mathbb{R}^{d}\right)} .
$$


Since $|G(x)|=\frac{C}{|x|^{d-2}}$ and $|\nabla G(x)|=\frac{C}{|x|^{d-1}}$ this is a particular case of well known fractional integration results for the Riesz potential $I_{\alpha} f=\frac{1}{\text {. }^{d-\alpha}} * f$ with $\alpha=2,1$, and we refer to [23, 33, for details. Here $L_{w}^{q}\left(\mathbb{R}^{d}\right)$ denotes the weak- $L^{q}$ space and coincides with the usual Lorentz space $L^{q, \infty}\left(\mathbb{R}^{d}\right)$.

As an immediate consequence we have the following integration by parts formula:

Proposition 3.1. Let $d \geq 3$ and $w \in L^{2 d /(d+2)}\left(\mathbb{R}^{d}\right)$. Then $\Phi=(-\Delta)^{-1} w=G * w$ satisfies $\Phi \in L^{2 d /(d-2)}\left(\mathbb{R}^{d}\right), \nabla \Phi \in L^{2}\left(\mathbb{R}^{d}\right)$, and

$$
\int_{\mathbb{R}^{d}}|\nabla \Phi|^{2} \mathrm{~d} x=\int_{\mathbb{R}^{d}} \Phi w \mathrm{~d} x=\iint_{\mathbb{R}^{d} \times \mathbb{R}^{d}} w(x) G(x-y) w(y) \mathrm{d} x \mathrm{~d} y .
$$

We shall use this later on with $w=u-v$ in order to control $\psi=G *(u-v)$.

Proof. Taking $p=\frac{2 d}{d+2} \in(1, d / 2)$ in (HLS-1) (HLS-3) we see that $\Phi \in L^{2 d /(d-2)}\left(\mathbb{R}^{d}\right)$ and $\nabla \Phi \in L^{2}\left(\mathbb{R}^{d}\right)$. Since $(2 d /(d+2))^{\prime}=2 d /(d-2)$ all the integrals in (3.1) are absolutely convergent and the last equality holds by Fubini's theorem. In order to retrieve the first equality we use approximation: if $w_{n} \in \mathcal{C}_{c}^{\infty}\left(\mathbb{R}^{d}\right)$ converges to $w$ in $L^{2 d /(d+2)}\left(\mathbb{R}^{d}\right)$ then by the HLS lemma $\Phi_{n} \rightarrow \Phi$ in $L^{2 d /(d-2)}\left(\mathbb{R}^{d}\right)$ and $\nabla \Phi_{n} \rightarrow \nabla \Phi$ in $L^{2}\left(\mathbb{R}^{d}\right)$. Since (3.1) holds for smooth $w_{n} \in \mathcal{C}_{c}^{\infty}$ with $-\Delta \Phi_{n}=w_{n}$ we conclude by letting $n \rightarrow \infty$.

Back to our energy functional, we begin with a fairly standard type of result [8, 16]:

Proposition 3.2 (Energy lower bound). Let $m \geq 1$ and $\mathcal{K}$ as in (2.2). The total free energy $\mathcal{E}$ is a proper functional on $\mathcal{K} \times \mathcal{K}$ and

$$
\inf _{\mathcal{K} \times \mathcal{K}} \mathcal{E}(u, v)>-\infty
$$

Moreover we have in every sub-levelset $\{\mathcal{E}(u, v) \leq R\}$ that

(i) gradient control: $\|\nabla \psi\|_{L^{2}\left(\mathbb{R}^{d}\right)} \leq C$

(ii) no concentration: if $m>1$ then

$$
\int_{\mathbb{R}^{d}}\left(u^{m}+v^{m}\right) \mathrm{d} x \leq C
$$

while if $m=1$ then

$$
\int_{\mathbb{R}^{d}}(u|\log u|+v|\log v|) \mathrm{d} x \leq C .
$$

(iii) mass confinement: $\int_{\mathbb{R}^{d}}|x|^{2}(u+v) \mathrm{d} x \leq C$, for some $C>0$ depending on $R>0$, the confining potentials, and $m$.

Proof. Choosing $u, v$ smooth and compactly supported it is clear that $\mathcal{E}(u, v)<\infty$ so $\mathcal{E}$ is proper.

$\mathbf{m}>1$ : (i)-(ii) immediately hold because each term in (1.3) is nonnegative. (iii) then follows by $\int_{\mathbb{R}^{d}}(u U+v V) \mathrm{d} x \leq \mathcal{E}(u, v)$ together with (2.1).

$\mathbf{m}=\mathbf{1}:$ if $\mathfrak{m}_{2}(\rho)=\int_{\mathbb{R}^{d}}|x|^{2} \rho \mathrm{d} x$ denotes the second moment let us first recall [16] the Carleman estimate

$$
\mathcal{H}(\rho) \geq-\int_{\mathbb{R}^{d}} \rho(\log \rho)^{-} \mathrm{d} x \geq-C\left(1+\mathfrak{m}_{2}(\rho)\right)^{\alpha}, \rho \in \mathcal{P},
$$


for some $C>0$ and $\alpha \in(0,1)$ depending on the dimension $d$ only. By (2.1) we have $\mathcal{E}_{\text {ext }}(u, v) \geq C_{1}\left(\mathfrak{m}_{2}(u)+\mathfrak{m}_{2}(v)\right)$, whence

$$
\begin{aligned}
C\left[\mathfrak{m}_{2}(u)+\right. & \left.\mathfrak{m}_{2}(v)-\left(1+\mathfrak{m}_{2}(u)\right)^{\alpha}-\left(1+\mathfrak{m}_{2}(v)\right)^{\alpha}\right] \\
& \leq \mathcal{H}(u)+\mathcal{H}(v)+\int_{\mathbb{R}^{d}}(u U+v V) \mathrm{d} x \leq \mathcal{E}(u, v) \leq C .
\end{aligned}
$$

Hence the second moments are bounded as in (iii). Then (i) and (ii) come immediately from (iii) and (3.2).

For fixed $z_{*}=\left(u_{*}, v_{*}\right) \in \mathcal{P} \times \mathcal{P}$, and given time step $h>0$ we set

$$
\mathcal{F}_{h}(z):=\frac{1}{2 h} d^{2}\left(z, z_{*}\right)+\mathcal{E}(z), \quad z=(u, v) \in \mathcal{K} \times \mathcal{K} .
$$

In order to define later a discrete sequence of approximate solutions using the JKO minimizing scheme, we collect here some properties of $\mathcal{F}_{h}$ and preliminary results.

Proposition 3.3 (Existence of minimizers). Fix $h>0$, and $z_{*}=\left(u_{*}, v_{*}\right) \in \mathcal{P} \times \mathcal{P}$. Then $\mathcal{F}_{h}$ admits a unique minimizer $z=(u, v) \in \mathcal{K} \times \mathcal{K}$.

Proof. By Proposition 3.2, $\mathcal{F}_{h}$ is bounded from below on $\mathcal{K} \times \mathcal{K}$, hence there is a minimizing sequence $z_{k}=\left(u_{k}, v_{k}\right)$ satisfying (i)-(iii) and $\left\{u_{k}, v_{k}\right\}_{k}$ are tight and uniformly integrable. By the Dunford-Pettis Theorem one may extract a subsequence such that

$$
u_{k} \rightarrow u \text { and } v_{k} \rightarrow v \quad \text { in } L^{1}\left(\mathbb{R}^{d}\right),
$$

and standard truncation arguments together with the uniform bounds on the second moments ensure that $u, v \in \mathcal{P}$. The weak $L^{1}$ lower semi-continuity (l.s.c.) of the squared Wasserstein distance, diffusive and potential energies are standard, in particular $u, v \in \mathcal{K}$. We prove in the appendix, Proposition 6.1 that the Dirichlet energy is lower semicontinuous with respect to weak $L^{1}\left(\mathbb{R}^{d}\right)$ convergence. Because $u_{k}-v_{k} \rightarrow u-v$ in $L^{1}\left(\mathbb{R}^{d}\right)$ we conclude here that $\mathcal{E}_{\mathrm{cpl}}(u, v) \leq \liminf _{k \rightarrow \infty} \mathcal{E}_{\mathrm{cpl}}\left(u_{k}, v_{k}\right)$, thus $u, v$ is a minimizer. Finally, the uniqueness result comes from the fact that the admissible set $\mathcal{K} \times \mathcal{K}$ is convex w.r.t. linear interpolation $z_{\theta}=(1-\theta) z_{0}+\theta z_{1}$ and that the total free energy is jointly strictly convex in $(u, v)$.

We remark that the squared distance term left aside in (3.3), the same line of argument would readily give existence of a global minimizer of the total free energy $\mathcal{E}$, which would result in the end in a least energy stationary weak solution $\underline{u}, \underline{v}$ to (1.1). Since our gradient flow system is driven by $\mathcal{E}$ one could expect long time convergence $u(t), v(t) \rightarrow \underline{u}, \underline{v}$ when $t \rightarrow \infty$ together with some convergence rates. However, the lack of displacement convexity prevents here from applying standard techniques [2, 11, 30] and this is beyond the scope of this paper. We refer to [6, 7] for related results on similar PNP models.

In the next section we shall derive the discrete Euler-Lagrange equations satisfied by the minimizers, which requires integration by parts as in (3.1). However, at this stage the minimizers only lie in $L^{m}\left(\mathbb{R}^{d}\right)$ if $m>1$ and $L^{1} \log L^{1}\left(\mathbb{R}^{d}\right)$ if $m=1$, and this manipulation is not justified. The discrete Euler-Lagrange equations are necessary to pass to the limit as the time step $h \rightarrow 0$ and to thereby obtain a solution to the PNP system. The remainder of this section is devoted to improving the regularity of the minimizers of the discrete functional.

The argument is based on the flow interchange technique of Matthes, McCann, and Savaré, [25], as implemented by Blanchet and Laurençot [10], as well as Laurençot and Matioc [22] and [9]. The idea of the flow interchange technique is that a known 
gradient flow is sufficiently close, generally first order close, to the one under study so that it may be used as an approximation with controllable error. We need to use this method twice, first to propagate the regularity of the minimizers and then to establish some smoothness of their spatial gradients. The characterization of gradient flow that is useful here is the so called Evolution Variational Inequality $(\mathrm{EVI})$ for a functional $\mathcal{F}$. Using the notation to follow, a flow $(\tilde{u}(t))$ is a gradient flow in the EVI sense, 1, 2, provided that

$$
\frac{1}{2} \frac{\mathrm{d}}{\mathrm{d} t} d_{W}^{2}(\tilde{u}(t), w)+\mathcal{F}(\tilde{u}(t)) \leq \mathcal{F}(w) \text { for all } w \in \mathcal{P}^{a c}\left(\mathbb{R}^{d}\right) \text { and a.e. } t>0 .
$$

Displacement convexity and the other detailed requirements for (3.4) to hold are discussed in the references just cited. For our purposes we note that (3.4) is valid for

(1) solutions of $\partial_{t} \tilde{u}=\Delta \tilde{u}$, the heat equation, with $\mathcal{F}=\mathcal{H}$, the Boltzmann entropy

$$
\mathcal{H}(\tilde{u})=\int_{\mathbb{R}^{d}} \tilde{u} \log \tilde{u} \mathrm{~d} x \text { and }
$$

(2) solutions of $\partial_{t} \tilde{u}=\Delta\left(\tilde{u}^{p}\right)$, the porous medium flow, with $\mathcal{F}=\mathcal{E}_{p}, 1<p<\infty$, given by the functional

$$
\mathcal{E}_{p}(\tilde{u}):=\frac{1}{p-1} \int_{\mathbb{R}^{d}} \tilde{u}^{p} \mathrm{~d} x .
$$

Proposition 3.4 (Discrete propagation of $L^{p}$ estimates). Let $m \geq 1, \lambda$ as in (2.13), and further assume that $u_{*}, v_{*} \in \mathcal{K} \cap L^{p}\left(\mathbb{R}^{d}\right)$ for some $p \in(1, \infty)$. If $0<h<h_{0}(p)=$ $\frac{1}{\lambda(p-1)}$ then the minimizer $(u, v)$ from Proposition 3.3 satisfies

$$
\|u\|_{L^{p}\left(\mathbb{R}^{d}\right)}^{p}+\|v\|_{L^{p}\left(\mathbb{R}^{d}\right)}^{p} \leq \frac{1}{1-\lambda(p-1) h}\left(\left\|u_{*}\right\|_{L^{p}\left(\mathbb{R}^{d}\right)}^{p}+\left\|v_{*}\right\|_{L^{p}\left(\mathbb{R}^{d}\right)}^{p}\right) .
$$

In this first use of the flow interchange, we simply use the solution of (3.8) below as variations in the minimum principle. Note that at this point the time step $h$ must be taken small in terms of $p$ for the minimizing problem to "see" the estimate. As a consequence there is no hope to retrieve an $L^{\infty}\left(\mathbb{R}^{d}\right)$ estimate at the discrete level for fixed $h$ directly from the limit $p \rightarrow \infty$ in (3.7), since $h<h_{0}(p)$ would require $h \rightarrow 0$. However, $u, v$ will be retrieved as some limit when $h \rightarrow 0$, so one can actually take $p$ arbitrarily large and the weak solutions will ultimately satisfy such an $L^{\infty}$ estimate. See the proof of Theorem 2 at the end of Section 5 for details.

Proof. For fixed $p \in(1, \infty)$ and $u_{*}, v_{*} \in \mathcal{K} \cap L^{p}$ consider the auxiliary Porous Media flows $\tilde{u}(t), \tilde{v}(t)$ defined by

$$
\begin{array}{ll}
\partial_{t} \tilde{u}=\Delta\left(\tilde{u}^{p}\right) \text { in }(0, \infty) \times \mathbb{R}^{d}, & \left.\tilde{u}\right|_{t=0}=u \text { in } \mathbb{R}^{d}, \\
\partial_{t} \tilde{v}=\Delta\left(\tilde{v}^{p}\right) \text { in }(0, \infty) \times \mathbb{R}^{d}, & \left.\tilde{v}\right|_{t=0}=v \text { in } \mathbb{R}^{d} .
\end{array}
$$

By standard results for the PME [34 we know that (i) these Cauchy problems are well posed and $\tilde{u}, \tilde{v} \in \mathcal{C}\left([0, \infty) ; L^{1}\left(\mathbb{R}^{d}\right)\right)$ remain probability measures, (ii) by $L^{1}-L^{\infty}$ smoothing $\tilde{u}(t), \tilde{v}(t) \in L^{\infty}\left(\mathbb{R}^{d}\right)$ for all $t>0$, and (iii) the second moments remain finite. As a consequence $\tilde{u}(t), \tilde{v}(t) \in \mathcal{K}$ are admissible for any $t>0$, and by Proposition 3.1 it will be no issue to integrate by parts in the coupling term.

Step 1: dissipation of the internal energy. We first claim that

$$
t>0: \quad \frac{\mathrm{d}}{\mathrm{d} t} \mathcal{E}_{\operatorname{diff}}(\tilde{u}, \tilde{v}) \leq 0,
$$


and we distinguish cases, $m=1$ being the most involved.

$m>1$ : By usual properties [34] of the PME all the $L^{q}\left(\mathbb{R}^{d}\right)$ norms are non-increasing along the PME flow, in particular $\mathcal{E}_{\text {diff }}(\tilde{u}, \tilde{v})=\frac{1}{m-1}\left(\|\tilde{u}\|_{L^{m}\left(\mathbb{R}^{d}\right)}^{m}+\|\tilde{v}\|_{L^{m}\left(\mathbb{R}^{d}\right)}^{m}\right)$ is nonincreasing in time.

$m=1$ : Assume first that $u$ is smooth and positive. Then by standard properties of the PME flow so is $\tilde{u}(t)$ for later times and, therefore, we get

$$
\frac{\mathrm{d}}{\mathrm{d} t}\left(\int_{\mathbb{R}^{d}} \tilde{u} \log \tilde{u} \mathrm{~d} x\right)=\int_{\mathbb{R}^{d}}(\log \tilde{u}) \Delta \tilde{u}^{p} \mathrm{~d} x=-\frac{4}{p} \int_{\mathbb{R}^{d}}\left|\nabla \tilde{u}^{p / 2}\right|^{2} \mathrm{~d} x \leq 0
$$

for $t>0$. Thus $t \mapsto \mathcal{H}(\tilde{u}(t))$ is nonincreasing.

If $u$ is not smooth and positive we first regularize it by running the heat equation for small times

$$
u_{k}=\Gamma_{1 / k} * u \underset{k \rightarrow \infty}{\rightarrow} u,
$$

where $\Gamma_{s}$ is the usual heat kernel at time $s$. Since the heat equation is the $\mathcal{H}$-gradient flow we have in particular $\mathcal{H}\left(u_{k}\right) \leq \mathcal{H}(u)$, and of course $u_{k}$ is positive and smooth. Denoting by $\tilde{u}_{k}(t)$ the solution of the corresponding PME-flow $\partial_{t} \tilde{u}_{k}=\Delta \tilde{u}_{k}^{p}$ starting from $u_{k}$, then the previous computation (3.10) shows that

$$
\forall t>0: \quad \mathcal{H}\left(\tilde{u}_{k}(t)\right) \leq \mathcal{H}\left(\tilde{u}_{k}(0)\right) \leq \mathcal{H}(u) .
$$

Since $u_{k} \rightarrow u$ in $L^{1}\left(\mathbb{R}^{d}\right)$, we get by standard $L^{1}$ contractivity of the PME that $\tilde{u}_{k}(t) \rightarrow \tilde{u}(t)$ in $L^{1}\left(\mathbb{R}^{d}\right)$ uniformly in $t \geq 0$ when $k \rightarrow \infty$, in particular weakly in $L^{1}\left(\mathbb{R}^{d}\right)$. By lower semi-continuity of $\mathcal{H}$ with respect to weak $L^{1}$ convergence we conclude that

$$
\forall t>0: \quad \mathcal{H}(\tilde{u}(t)) \leq \liminf _{k \rightarrow \infty} \mathcal{H}\left(\tilde{u}_{k}(t)\right) \leq \mathcal{H}(u) .
$$

Finally, by uniqueness of solutions to the PME flow with $\tilde{u}(0)=u$ we conclude that $t \mapsto \mathcal{H}(\tilde{u}(t))$ is monotone nonincreasing, and similarly arguing for $v$ entails (3.9) as claimed.

Step 2: the remaining terms. Arguing by approximation [34 the potential energy is easily controlled for $t>0$ as

$$
\begin{aligned}
\frac{\mathrm{d}}{\mathrm{d} t} \mathcal{E}_{\text {ext }}(\tilde{u}, \tilde{v}) & =\frac{\mathrm{d}}{\mathrm{d} t} \int_{\mathbb{R}^{d}}(\tilde{u} U+\tilde{v} V) \mathrm{d} x=\int_{\mathbb{R}^{d}}\left[\left(\Delta \tilde{u}^{p}\right) U+\left(\Delta \tilde{v}^{p}\right) V\right] \mathrm{d} x \\
& =\int_{\mathbb{R}^{d}}\left[\tilde{u}^{p} \Delta U+\tilde{v}^{p} \Delta V\right] \mathrm{d} x \leq \lambda \int_{\mathbb{R}^{d}}\left(\tilde{u}^{p}+\tilde{v}^{p}\right) \mathrm{d} x .
\end{aligned}
$$

For the coupling term, let $\tilde{\psi}(t)=G *(\tilde{u}-\tilde{v})(t)$ and observe that for $t>0$ we have $\partial_{t} \tilde{\psi}=\partial_{t}\left[(-\Delta)^{-1}(\tilde{u}-\tilde{v})\right]=(-\Delta)^{-1}\left[\partial_{t}(\tilde{u}-\tilde{v})\right]=-\left(\tilde{u}^{p}-\tilde{v}^{p}\right)$. Since $\tilde{u}(t), \tilde{v}(t) \in$ $L^{\infty}\left(\mathbb{R}^{d}\right)$ for $t>0$ we can legitimately integrate by parts

$$
\begin{aligned}
\frac{\mathrm{d}}{\mathrm{d} t} \mathcal{E}_{\text {cpl }}(\tilde{u}, \tilde{v}) & =\frac{\mathrm{d}}{\mathrm{d} t}\left(\frac{1}{2} \int_{\mathbb{R}^{d}}|\nabla \tilde{\psi}|^{2} \mathrm{~d} x\right) \\
& =\int_{\mathbb{R}^{d}}(-\Delta \tilde{\psi}) \partial_{t} \tilde{\psi} \mathrm{d} x=-\int_{\mathbb{R}^{d}}(\tilde{u}-\tilde{v}) \cdot\left(\tilde{u}^{p}-\tilde{v}^{p}\right) \mathrm{d} x \leq 0 .
\end{aligned}
$$

Note that, due to $\|\nabla \tilde{\psi}\|_{L^{2}\left(\mathbb{R}^{d}\right)}=\left\|\nabla(-\Delta)^{-1}(\tilde{u}-\tilde{v})\right\|_{L^{2}\left(\mathbb{R}^{d}\right)} \approx\|\tilde{u}-\tilde{v}\|_{H^{-1}\left(\mathbb{R}^{d}\right)}$, this is the well-known $H^{-1}$ contraction property of the PME flow, see [34]. 
${ }^{14}$ As for the Wasserstein term, note that $\tilde{u}, \tilde{v}$ are respective gradient flows of the functional $\mathcal{E}_{p}$, (3.6), so from (3.4),

$$
\begin{aligned}
& \frac{1}{2 h} \frac{\mathrm{d}}{\mathrm{d} t}\left[d_{W}\left(\tilde{u}, u_{*}\right)^{2}+d_{W}\left(\tilde{v}, v_{*}\right)^{2}\right] \\
& \quad \leq \frac{1}{(p-1) h} \int_{\mathbb{R}^{d}}\left(u_{*}^{p}-\tilde{u}^{p}\right) \mathrm{d} x+\frac{1}{(p-1) h} \int_{\mathbb{R}^{d}}\left(v_{*}^{p}-\tilde{v}^{p}\right) \mathrm{d} x .
\end{aligned}
$$

Step 3: dissipation inequality. Gathering (3.9), (3.11), (3.12), and (3.13), we get the total dissipation inequality

$$
\begin{aligned}
\mathcal{D}(t):=\frac{\mathrm{d}}{\mathrm{d} t} \mathcal{F}_{h}(\tilde{u}, \tilde{v}) \leq & \frac{1}{(p-1) h} \int_{\mathbb{R}^{d}}\left(u_{*}^{p}+v_{*}^{p}\right) \mathrm{d} x-\frac{1}{(p-1) h} \int_{\mathbb{R}^{d}}\left(\tilde{u}^{p}+\tilde{v}^{p}\right) \mathrm{d} x \\
& +\lambda \int_{\mathbb{R}^{d}}\left(\tilde{u}^{p}+\tilde{v}^{p}\right) \mathrm{d} x=: \mathcal{A}(t)
\end{aligned}
$$

for small $t>0$. Because $(\tilde{u}(0), \tilde{v}(0))=(u, v)$ is a minimizer we must have $D(t) \geq 0$ at least for a time sequence $t_{n} \searrow 0$, otherwise $(\tilde{u}(t), \tilde{v}(t))$ would be a strictly better competitor for small $t>0$. If $0<h<h_{0}=\frac{1}{\lambda(p-1)}$ we have $1-\lambda(p-1) h>0$ and $\mathcal{A}\left(t_{n}\right) \geq \mathcal{D}\left(t_{n}\right) \geq 0$ can be rearranged as

$$
\int_{\mathbb{R}^{d}}\left[\tilde{u}^{p}\left(t_{n}\right)+\tilde{v}^{p}\left(t_{n}\right)\right] \mathrm{d} x \leq \frac{1}{1-\lambda(p-1) h} \int_{\mathbb{R}^{d}}\left(u_{*}^{p}+v_{*}^{p}\right) \mathrm{d} x .
$$

Our statement follows by finally letting $t_{n} \searrow 0$ in (3.14), recalling that $(\tilde{u}(t), \tilde{v}(t)) \rightarrow$ $(u, v)$ in $L^{1}\left(\mathbb{R}^{d}\right)$ when $t \rightarrow 0$.

We shall also need a further regularity result for the gradient of $(u, v)$. The use of the flow interchange in this estimate is very similar to its use in [10] for the critical parabolic-parabolic Keller-Segel model.

Proposition 3.5 (Discrete gradient estimate). For $m \geq 1, d \geq 3$, and any $h>0$, fix $z_{*}=\left(u_{*}, v_{*}\right) \in \mathcal{P} \times \mathcal{P}$ and let $z=(u, v) \in \mathcal{K} \times \mathcal{K}$ be the unique minimizer from Proposition 3.3. Then

$$
\begin{aligned}
& \left\|\nabla\left(u^{m / 2}\right)\right\|_{L^{2}\left(\mathbb{R}^{d}\right)}^{2}+\left\|\nabla\left(v^{m / 2}\right)\right\|_{L^{2}\left(\mathbb{R}^{d}\right)}^{2} \\
& \quad \leq C\left[1+\frac{\mathcal{H}\left(u_{*}\right)-\mathcal{H}(u)}{h}+\frac{\mathcal{H}\left(v_{*}\right)-\mathcal{H}(v)}{h}\right]
\end{aligned}
$$

for some $C>0$ independent of $h>0$ and $z_{*}$.

Proof. We use a second flow interchange with $\tilde{u}(t), \tilde{v}(t)$ now defined by

$$
\partial_{t} \tilde{u}-\Delta \tilde{u}=0 \text { in }(0, \infty) \times \mathbb{R}^{d},\left.\quad \tilde{u}\right|_{t=0}=u \text { in } \mathbb{R}^{d}
$$

and

$$
\partial_{t} \tilde{v}-\Delta \tilde{v}=0 \text { in }(0, \infty) \times \mathbb{R}^{d},\left.\quad \tilde{v}\right|_{t=0}=v \text { in } \mathbb{R}^{d} .
$$

Step 1: dissipation inequality. We first note that classical properties of the heat equation and $\tilde{u}(0), \tilde{v}(0) \in \mathcal{K}$ guarantee $\tilde{u}(t), \tilde{v}(t) \in \mathcal{K}$ for all $t>0$. Let $\tilde{\psi}:=$ $G *(\tilde{u}-\tilde{v})$. Then it is easy to check that $\partial_{t} \tilde{\psi}=\Delta \tilde{\psi}$ as well. Since the pair $(u, v)$ is a minimizer and has finite energy we have in particular $\nabla \tilde{\psi}(0)=\nabla \psi \in L^{2}\left(\mathbb{R}^{d}\right)$, whence by standard properties of the heat equation $\nabla \tilde{\psi}(t) \in L^{2}\left(\mathbb{R}^{d}\right)$ and

$$
\frac{\mathrm{d}}{\mathrm{d} t}\|\nabla \tilde{\psi}(t)\|_{L^{2}\left(\mathbb{R}^{d}\right)}^{2} \leq 0
$$

for all $t>0$. Since $\tilde{u}, \tilde{v}$ are positive and smooth for all $t>0$ we may differentiate and integrate by parts as 
$\mathbf{m}>1$ :

$$
\begin{aligned}
& \frac{\mathrm{d}}{\mathrm{d} t} \mathcal{E}(\tilde{u}, \tilde{v}) \\
& =\frac{m}{m-1} \int_{\mathbb{R}^{d}}\left(\tilde{u}^{m-1} \Delta \tilde{u}+\tilde{v}^{m-1} \Delta \tilde{v}\right) \mathrm{d} x+\int_{\mathbb{R}^{d}}(U \Delta \tilde{u}+V \Delta \tilde{v}) \mathrm{d} x \\
& \quad+\frac{\mathrm{d}}{\mathrm{d} t}\left(\frac{1}{2}\|\nabla \tilde{\psi}(t)\|_{L^{2}\left(\mathbb{R}^{d}\right)}^{2}\right) \\
& \leq-\frac{4}{m} \int_{\mathbb{R}^{d}}\left(\left|\nabla \tilde{u}^{\frac{m}{2}}\right|^{2}+\left|\nabla \tilde{v}^{\frac{m}{2}}\right|^{2}\right) \mathrm{d} x+\int_{\mathbb{R}^{d}}(\tilde{u} \Delta U+\tilde{v} \Delta V) \mathrm{d} x \\
& \leq-\frac{4}{m} \int_{\mathbb{R}^{d}}\left(\left|\nabla \tilde{u}^{\frac{m}{2}}\right|^{2}+\left|\nabla \tilde{v}^{\frac{m}{2}}\right|^{2}\right) \mathrm{d} x+\|\Delta U\|_{L^{\infty}\left(\mathbb{R}^{d}\right)}+\|\Delta V\|_{L^{\infty}\left(\mathbb{R}^{d}\right)}
\end{aligned}
$$

and similarly

$\mathbf{m}=\mathbf{1}$ :

$$
\frac{\mathrm{d}}{\mathrm{d} t} \mathcal{E}(\tilde{u}, \tilde{v}) \leq-4 \int_{\mathbb{R}^{d}}\left(\left|\nabla \tilde{u}^{\frac{1}{2}}\right|^{2}+\left|\nabla \tilde{v}^{\frac{1}{2}}\right|^{2}\right) \mathrm{d} x+\|\Delta U\|_{L^{\infty}\left(\mathbb{R}^{d}\right)}+\|\Delta V\|_{L^{\infty}\left(\mathbb{R}^{d}\right)} .
$$

Here (2.1) is used in the third inequality of both (3.18) and (3.19).

For $m>1$, the above term $\frac{4}{m} \int_{\mathbb{R}^{d}}\left|\nabla \tilde{\rho}^{\frac{m}{2}}\right|^{2} \mathrm{~d} x$ in (3.18) (dissipation of $\mathcal{E}_{m}(\rho)=$ $\frac{1}{m-1} \int_{\mathbb{R}^{d}} \rho^{m} \mathrm{~d} x$ along $\left.\partial_{t} \rho=\Delta \rho\right)$ corresponds to the usual Fisher information $-4 \int_{\mathbb{R}^{d}}\left|\nabla \rho^{\frac{1}{2}}\right|^{2} \mathrm{~d} x=$ $\frac{\mathrm{d}}{\mathrm{d} t} \mathcal{H}(\rho)$ in the linear diffusion case (3.19), and enjoys a formal continuity when $m \searrow 1$. Comparing with (3.10) for $p=m$ we also see that the dissipation of $\mathcal{H}$ along the $\mathcal{E}_{m}$-flow equals the dissipation of $\mathcal{E}_{m}$ along the $\mathcal{H}$-flow, which is in fact the cornerstone of this flow interchange technique.

Let

$$
\mathcal{D}(t):=\frac{4}{m}\left(\left\|\nabla \tilde{u}^{\frac{m}{2}}(t)\right\|_{L^{2}\left(\mathbb{R}^{d}\right)}^{2}+\left\|\nabla \tilde{v}^{\frac{m}{2}}(t)\right\|_{L^{2}\left(\mathbb{R}^{d}\right)}^{2}\right) .
$$

Integrating (3.18) or (3.19) from 0 to $t>0$ we get in both cases

$$
\mathcal{E}(\tilde{u}(t), \tilde{v}(t))-\mathcal{E}\left(u_{*}, v_{*}\right) \leq 2 \lambda t-\int_{0}^{t} \mathcal{D}(s) \mathrm{d} s,
$$

with $\lambda$ defined in (2.13). Because (3.16)-(3.17) are respective $\mathcal{H}$-gradient flows, we again appeal to (3.4) to obtain

$$
\begin{aligned}
\frac{1}{2} \frac{\mathrm{d}}{\mathrm{d} t} d^{2}\left(\tilde{z}(t), z_{*}\right) & =\frac{1}{2} \frac{\mathrm{d}}{\mathrm{d} t}\left[d_{W}^{2}\left(\tilde{u}(t), u_{*}\right)+d_{W}^{2}\left(\tilde{v}(t), v_{*}\right)\right] \\
& \leq \mathcal{H}\left(u_{*}\right)-\mathcal{H}(\tilde{u}(t))+\mathcal{H}\left(v_{*}\right)-\mathcal{H}(\tilde{v}(t)) .
\end{aligned}
$$

Integrating again and using the monotonicity of $s \searrow \mathcal{H}(\tilde{u}(s))$ and $s \searrow \mathcal{H}(\tilde{v}(s))$ along the flow with $\tilde{z}(0)=z$ gives

$$
\frac{1}{2 h}\left[d^{2}\left(\tilde{z}(t), z_{*}\right)-d^{2}\left(z, z_{*}\right)\right] \leq \frac{t}{h}\left[\mathcal{H}\left(u_{*}\right)-\mathcal{H}(\tilde{u}(t))+\mathcal{H}\left(v_{*}\right)-\mathcal{H}(\tilde{v}(t))\right] .
$$

Since $z$ is a minimizer we have by (3.3) and (3.20) that for small $t>0$

$$
\begin{aligned}
0 & \leq \mathcal{F}_{h}(\tilde{z}(t))-\mathcal{F}_{h}(z) \\
& \leq \frac{t}{h}\left[\mathcal{H}\left(u_{*}\right)-\mathcal{H}(\tilde{u}(t))+\mathcal{H}\left(v_{*}\right)-\mathcal{H}(\tilde{v}(t))\right]+2 \lambda t-\int_{0}^{t} D(s) \mathrm{d} s,
\end{aligned}
$$

which we reformulate as

$$
\frac{1}{t} \int_{0}^{t} \mathcal{D}(s) \mathrm{d} s \leq 2 \lambda+\frac{\mathcal{H}\left(u_{*}\right)-\mathcal{H}(\tilde{u}(t))}{h}+\frac{\mathcal{H}\left(v_{*}\right)-\mathcal{H}(\tilde{v}(t))}{h} .
$$


Step 2: the limit $t \rightarrow 0$. If

$$
D_{1}(t, x):=\frac{1}{t} \int_{0}^{t} \tilde{u}^{\frac{m}{2}}(s, x) \mathrm{d} s, \quad D_{2}(t, x):=\frac{1}{t} \int_{0}^{t} \tilde{v}^{\frac{m}{2}}(s, x) \mathrm{d} s,
$$

we first note that $\tilde{u}, \tilde{v} \in \mathcal{C}\left([0, \infty) ; L^{m}\left(\mathbb{R}^{d}\right)\right)$ as solutions of the heat equation with initial data in $L^{m}\left(\mathbb{R}^{d}\right)$, so that $D_{1}, D_{2} \in \mathcal{C}\left([0, \infty) ; L^{2}\left(\mathbb{R}^{d}\right)\right)$. As a consequence $D_{1}(t) \rightarrow D_{1}(0)=u^{\frac{m}{2}}$ and $D_{2}(t) \rightarrow D_{2}(0)=v^{\frac{m}{2}}$ in $L^{2}\left(\mathbb{R}^{d}\right)$ when $t \downarrow 0$. By (3.21) we find that $\nabla D_{1}(t)$ and $\nabla D_{2}(t)$ are bounded in $L^{2}\left(\mathbb{R}^{d}\right)$ and converge at least in $\mathcal{D}^{\prime}\left(\mathbb{R}^{d}\right)$ to $\nabla\left(u^{\frac{m}{2}}\right)$ and $\nabla\left(v^{\frac{m}{2}}\right)$ when $t \rightarrow 0$. Consequently, $\nabla\left(u^{\frac{m}{2}}\right), \nabla\left(v^{\frac{m}{2}}\right) \in L^{2}\left(\mathbb{R}^{d}\right)$ and our statement follows from

$$
\begin{aligned}
\left\|\nabla\left(u^{\frac{m}{2}}\right)\right\|_{L^{2}\left(\mathbb{R}^{d}\right)}^{2} & +\left\|\nabla\left(v^{\frac{m}{2}}\right)\right\|_{L^{2}\left(\mathbb{R}^{d}\right)}^{2} \\
& \leq \liminf _{t \rightarrow 0}\left\|\nabla D_{1}(t)\right\|_{L^{2}\left(\mathbb{R}^{d}\right)}^{2}+\liminf _{t \rightarrow 0}\left\|\nabla D_{2}(t)\right\|_{L^{2}\left(\mathbb{R}^{d}\right)}^{2} \\
& \leq \frac{m}{4} \liminf _{t \rightarrow 0} \frac{1}{t} \int_{0}^{t} \mathcal{D}(s) d s \\
& \leq C\left(1+\frac{\mathcal{H}\left(u_{*}\right)-\mathcal{H}(u)}{h}+\frac{\mathcal{H}\left(v_{*}\right)-\mathcal{H}(v)}{h}\right) .
\end{aligned}
$$

The last inequality comes from the limit $t \searrow 0$ in (3.21) with strong convergence $(\tilde{u}(t), \tilde{v}(t)) \rightarrow(u, v)$, e.g. in $L^{1} \cap L^{r_{0}}\left(\mathbb{R}^{d}\right)$, and suitable continuity of $\rho \mapsto \mathcal{H}(\rho)$.

\section{Minimizing SCheme And DisCRete estimates}

In this section, we shall construct a family of time-discrete approximate solutions using the JKO method, also known as the variational minimizing movement scheme. A priori estimates for the set of discrete solutions are necessary to allow us to deduce the existence of a time-continuous limit curve.

Fix an initial datum $z^{0}=\left(u^{0}, v^{0}\right)$ as in (2.3) and some time step $h>0$. Setting $z_{h}^{(0)}=z^{0}$, Proposition 3.3 allows us to define a sequence $z_{h}^{(n)}=\left(u_{h}^{(n)}, v_{h}^{(n)}\right) \in \mathcal{K} \times \mathcal{K}$ recursively as

$$
z_{h}^{(n+1)}:=\text { the unique minimizer } z \text { of } \mathcal{F}_{h} \text { with } z_{*}=z_{h}^{(n)}=\left(u_{h}^{(n)}, v_{h}^{(n)}\right)
$$

and a corresponding piecewise-constant interpolation $t \in[0, \infty) \mapsto z_{h}(t)$ as

$$
z_{h}(t)=z_{h}^{(n)} \text { for } n h \leq t<(n+1) h .
$$

The rest of this section is devoted to collecting the suitable a priori estimates on $z_{h}$ suitable to pass to the limit in $h \searrow 0$.

It is now standard to get the discrete energy monotonicity as in [16] that

$$
\forall n \geq 0: \quad \mathcal{E}\left(z_{h}^{(n+1)}\right) \leq \mathcal{E}\left(z_{h}^{(n)}\right)
$$

inasmuch as $z_{h}^{(n)}$ is a competitor in the search for $z_{h}^{(n+1)}$. At the continuous level this reads as

$$
\mathcal{E}\left(z_{h}\left(t_{2}\right)\right) \leq \mathcal{E}\left(z_{h}\left(t_{1}\right)\right) \leq \mathcal{E}\left(z^{0}\right) \quad \text { for all } \quad 0 \leq t_{1} \leq t_{2} .
$$

Proposition 4.1. The total square distance and approximate Hölder estimates

$$
\begin{gathered}
\frac{1}{2 h} \sum_{n \geq 0} d^{2}\left(z_{h}^{(n)}, z_{h}^{(n+1)}\right) \leq \mathcal{E}\left(z^{0}\right)-\inf _{\mathcal{K} \times \mathcal{K}} \mathcal{E}, \\
\forall 0 \leq t_{1} \leq t_{2}, \quad d\left(z_{h}\left(t_{1}\right), z_{h}\left(t_{2}\right)\right) \leq C\left|t_{2}-t_{1}+h\right|^{\frac{1}{2}},
\end{gathered}
$$

hold for some $C>0$ independent of $h>0$. 
Proof. Note that since $u^{0}, v^{0} \in L^{1}\left(\mathbb{R}^{d}\right) \cap L^{2 d /(d+1)}\left(\mathbb{R}^{d}\right)$ we have in particular $u^{0}-v^{0} \underline{\underline{\epsilon}}$ $L^{2 d /(d+2)}\left(\mathbb{R}^{d}\right)$. By Proposition 3.1 we have $\nabla \psi^{0}=\nabla G *\left(u^{0}-v^{0}\right) \in L^{2}\left(\mathbb{R}^{d}\right)$ and $u^{0}, v^{0}$ has therefore finite energy. We also recall from Proposition 3.2 that $\inf _{\mathcal{K} \times \mathcal{K}} \mathcal{E}>-\infty$, hence the right-hand side in (4.1) is finite (and of course independent of $h>0$ ). The rest of the argument is by now very classical and we refer to [16].

Proposition 4.2. The piecewise constant interpolation satisfies

$$
\begin{aligned}
& m>1: \sup _{t \geq 0} \int_{\mathbb{R}^{d}}\left(u_{h}^{m}(t)+v_{h}^{m}(t)\right) \mathrm{d} x \leq C \\
& m=1: \sup _{t \geq 0} \int_{\mathbb{R}^{d}}\left(u_{h}(t)\left|\log u_{h}(t)\right|+v_{h}(t)\left|\log v_{h}(t)\right|\right) \mathrm{d} x \leq C
\end{aligned}
$$

and

$$
\sup _{t \geq 0} \int_{\mathbb{R}^{d}}|x|^{2}\left(u_{h}(t)+v_{h}(t)\right) \mathrm{d} x \leq C
$$

uniformly in $h>0$.

Proof. By energy monotonicity we have $\sup _{n \geq 0} \mathcal{E}\left(u_{h}^{(n)}, v_{h}^{(n)}\right) \leq \mathcal{E}\left(u^{0}, v^{0}\right)<\infty$, which by Proposition 3.2 bounds the internal energy and the second moments uniformly in $h, n$ for the discrete sequence. This property extends to the interpolation $u_{h}(t), v_{h}(t)$.

In addition to the uniform control in Proposition 4.2, we also have

Proposition 4.3 (Continuous $L^{p}$ estimate). In addition to (2.3) assume that the initial data $u^{0}, v^{0} \in L^{p}\left(\mathbb{R}^{d}\right)$ for some $p \in(1, \infty)$, and let $\lambda$ as in (2.13). Then for $h<h_{0}(p)=\frac{1}{\lambda(p-1)}$ sufficiently small we have

$$
\forall t \geq 0: \quad\left\|u_{h}(t)\right\|_{L^{p}\left(\mathbb{R}^{d}\right)}+\left\|v_{h}(t)\right\|_{L^{p}\left(\mathbb{R}^{d}\right)} \leq C e^{\lambda t}\left(\left\|u^{0}\right\|_{L^{p}\left(\mathbb{R}^{d}\right)}+\left\|v^{0}\right\|_{L^{p}\left(\mathbb{R}^{d}\right)}\right)
$$

for some $C>0$ independent of $t, p, h$, and the initial data.

Proof. Fix any $t>0$, let $k=\lfloor t / h\rfloor$, and recall that $u_{h}(t)=u_{h}^{(k)}$. By induction we immediately get from Proposition 3.4

$$
\left\|u_{h}(t)\right\|_{L^{p}\left(\mathbb{R}^{d}\right)}^{p}+\left\|v_{h}(t)\right\|_{L^{p}\left(\mathbb{R}^{d}\right)}^{p} \leq\left(\frac{1}{1-\lambda(p-1) h}\right)^{\lfloor t / h\rfloor}\left(\left\|u^{0}\right\|_{L^{p}\left(\mathbb{R}^{d}\right)}^{p}+\left\|v^{0}\right\|_{L^{p}\left(\mathbb{R}^{d}\right)}^{p}\right) .
$$

For small $h>0$ this easily gives

$$
\left\|u_{h}(t)\right\|_{L^{p}\left(\mathbb{R}^{d}\right)}+\left\|v_{h}(t)\right\|_{L^{p}\left(\mathbb{R}^{d}\right)} \leq C e^{\lambda \frac{p-1}{p} t}\left(\left\|u^{0}\right\|_{L^{p}\left(\mathbb{R}^{d}\right)}+\left\|v^{0}\right\|_{L^{p}\left(\mathbb{R}^{d}\right)}\right)
$$

for some universal $C>0$. Since $e^{\lambda \frac{p-1}{p} t} \leq e^{\lambda t}$ the proof is complete.

Proposition 4.4 (Approximate Euler-Lagrange equations). Fix $m \geq 1$. Let $\nabla q^{(n)}$ and $\nabla r^{(n)}$ be the optimal transport maps

$$
u_{h}^{(n+1)}=\left(\nabla q^{(n)}\right)_{\#} u_{h}^{(n)} \quad \text { and } \quad v_{h}^{(n+1)}=\left(\nabla r^{(n)}\right)_{\#} v_{h}^{(n)}
$$


in Brenier's Theorem 1, and $\psi_{h}^{(n)}=G *\left(u_{h}^{(n)}-v_{h}^{(n)}\right)$. Then for any vector-field $\zeta \in \mathcal{C}_{c}^{\infty}\left(\mathbb{R}^{d} ; \mathbb{R}^{d}\right)$, we have that

$$
\begin{aligned}
& \frac{1}{h} \int_{\mathbb{R}^{d}}\left\langle\nabla q^{(n)}-\mathrm{Id}, \zeta \circ \nabla q^{(n)}\right\rangle u_{h}^{(n)} \mathrm{d} x \\
& =\int_{\mathbb{R}^{d}}\left(u_{h}^{(n+1)}\right)^{m} \operatorname{div} \zeta \mathrm{d} x-\int_{\mathbb{R}^{d}} u_{h}^{(n+1)}\langle\nabla U, \zeta\rangle \mathrm{d} x-\int_{\mathbb{R}^{d}} u_{h}^{(n+1)}\left\langle\nabla \psi_{h}^{(n+1)}, \zeta\right\rangle \mathrm{d} x,
\end{aligned}
$$

and

$$
\begin{aligned}
& \frac{1}{h} \int_{\mathbb{R}^{d}}\left\langle\nabla r^{(n)}-\mathrm{Id}, \zeta \circ \nabla r^{(n)}\right\rangle v_{h}^{(n)} \mathrm{d} x \\
& =\int_{\mathbb{R}^{d}}\left(v_{h}^{(n+1)}\right)^{m} \operatorname{div} \zeta \mathrm{d} x-\int_{\mathbb{R}^{d}} v_{h}^{(n+1)}\langle\nabla V, \zeta\rangle \mathrm{d} x+\int_{\mathbb{R}^{d}} v_{h}^{(n+1)}\left\langle\nabla \psi_{h}^{(n+1)}, \zeta\right\rangle \mathrm{d} x .
\end{aligned}
$$

Proof. In order to simplify notations, we write below $u_{*}=u_{h}^{(n)}, u=u_{h}^{(n+1)}, v_{*}=$ $v_{h}^{(n)}, v=v_{h}^{(n+1)}$, and $\psi=\psi_{h}^{(n+1)}=G *\left[u_{h}^{(n+1)}-v_{h}^{(n+1)}\right]$. Fix an arbitrary vector-field $\zeta \in \mathcal{C}_{c}^{\infty}\left(\mathbb{R}^{d}, \mathbb{R}^{d}\right)$. For $\varepsilon \in[-\delta, \delta]$, let $\Phi_{\varepsilon}(x)$ be the associated $\varepsilon$-flow (i-e $d \Phi_{\varepsilon} / d \varepsilon=$ $\zeta\left(\Phi_{\varepsilon}\right)$ and $\Phi_{0}=\mathrm{Id}$ ), and let us consider the perturbation (of domain)

$$
u_{\varepsilon}:=\left(\Phi_{\varepsilon}\right)_{\#} u, \quad z_{\varepsilon}:=\left(u_{\varepsilon}, v\right) .
$$

Since $\left.z\right|_{\varepsilon=0}=z$ is a minimizer, computing the first variation $\frac{d}{d \varepsilon}\left(\mathcal{F}_{h}\left(z_{\varepsilon}\right)\right)_{\varepsilon=0}=0$ will classically give (4.5). Similarly considering $v_{\varepsilon}=\left(\Phi_{\varepsilon}\right)_{\#} v$ and $z_{\varepsilon}=\left(u, v_{\varepsilon}\right)$ will produce (4.6).

More precisely, differentiating the Wasserstein distance squared, the confining potential, and the diffusive energy are by now classical computations [2]. However, differentiating the coupling energy is quite delicate here: because we have to consider separate horizontal and vertical perturbations the nonscalar nature of the problem induces a loss of symmetry. Formally the result should follow from

$$
\int_{\mathbb{R}^{d}}\left|\nabla \psi_{\varepsilon}\right|^{2} \mathrm{~d} x=\int_{\mathbb{R}^{d}} \psi_{\varepsilon}\left(u_{\varepsilon}-v\right) \mathrm{d} x=\iint_{\mathbb{R}^{d} \times \mathbb{R}^{d}}\left[u_{\varepsilon}-v\right](x) G(x-y)\left[u_{\varepsilon}-v\right](y) \mathrm{d} x \mathrm{~d} y
$$

and the classical computations for interaction energies, see [35. But because we consider two components independently it might happen that $\nabla \psi_{\varepsilon} \notin L^{2}\left(\mathbb{R}^{d}\right)$ even though $\nabla \psi \in L^{2}\left(\mathbb{R}^{d}\right)$, and the above integration by parts might not be legitimate. Moreover since $\nabla G$ is more singular than $G$ itself, differentiating with respect to $\varepsilon$ requires some extra regularity. This can actually be made rigorous using the propagation of the initial regularity as follows. Since the initial datum $u^{0}, v^{0} \in$ $L^{2 d /(d+1)}\left(\mathbb{R}^{d}\right)$ and the time step is small enough, we have by Proposition 3.4 that $u, v \in L^{1}\left(\mathbb{R}^{d}\right) \cap L^{2 d /(d+1)}\left(\mathbb{R}^{d}\right)$, and in particular $u, u_{\varepsilon}, v \in L^{2 d /(d+2)}\left(\mathbb{R}^{d}\right)$. Using Proposition 3.1 we can therefore integrate by parts and expand with $u_{\varepsilon}=\left(\Phi_{\varepsilon}\right)_{\#} u$

$$
\begin{aligned}
& \int_{\mathbb{R}^{d}}\left|\nabla \psi_{\varepsilon}\right|^{2} \mathrm{~d} x=\iint_{\mathbb{R}^{d} \times \mathbb{R}^{d}} u(x) G\left(\Phi_{\varepsilon}(x)-\Phi_{\varepsilon}(y)\right) u(y) \mathrm{d} x \mathrm{~d} y \\
& \quad-2 \iint_{\mathbb{R}^{d} \times \mathbb{R}^{d}} u(x) G\left(\Phi_{\varepsilon}(x)-y\right) v(y) \mathrm{d} x \mathrm{~d} y+\text { terms independent of } \varepsilon
\end{aligned}
$$

where the last equality follows by definition of the pushforward $u_{\varepsilon}=\left(\Phi_{\varepsilon}\right)_{\#} u$. In order to differentiate under the integral sign we only need $L^{1}\left(\mathbb{R}^{d} \times \mathbb{R}^{d}\right)$ bounds such 
that

$$
\begin{array}{r}
\iint_{\mathbb{R}^{d} \times \mathbb{R}^{d}} u(x)\left|\left\langle\nabla G\left(\Phi_{\varepsilon}(x)-\Phi_{\varepsilon}(y)\right), \zeta \circ \Phi_{\varepsilon}(x)-\zeta \circ \Phi_{\varepsilon}(y)\right\rangle\right| u(y) \mathrm{d} x \mathrm{~d} y \leq C, \\
\iint_{\mathbb{R}^{d} \times \mathbb{R}^{d}} u(x)\left|\left\langle\nabla G\left(\Phi_{\varepsilon}(x)-y\right), \zeta \circ \Phi_{\varepsilon}(x)\right\rangle\right| v(y) \mathrm{d} x \mathrm{~d} y \leq C,
\end{array}
$$

uniformly as $\varepsilon \rightarrow 0$. Because $\Phi_{\varepsilon}$ is close to Id for small $\varepsilon, \zeta \in \mathcal{C}_{0}^{\infty}\left(\mathbb{R}^{d}\right)$, and

$$
|\nabla G(x-y)| \leq \frac{C}{|x-y|^{d-1}},
$$

this simply amounts to controlling

$$
\begin{aligned}
& \iint_{\mathbb{R}^{d} \times \mathbb{R}^{d}} u(x) \frac{1}{|x-y|^{d-1}} u(y) \mathrm{d} x \mathrm{~d} y \leq C, \\
& \iint_{\mathbb{R}^{d} \times \mathbb{R}^{d}} u(x) \frac{1}{|x-y|^{d-1}} v(y) \mathrm{d} x \mathrm{~d} y \leq C,
\end{aligned}
$$

which is valid by (HLS-3) with $p=2 d /(d+1)$ and $u, v \in L^{2 d /(d+1)}\left(\mathbb{R}^{d}\right)$. As a consequence we can legitimately compute with $\Phi_{0}=\mathrm{Id}$

$$
\begin{aligned}
\frac{\mathrm{d}}{\mathrm{d} \varepsilon}\left(\int_{\mathbb{R}^{d}}\left|\nabla \psi_{\varepsilon}\right|^{2} \mathrm{~d} x\right)_{\varepsilon=0}= & \iint_{\mathbb{R}^{d} \times \mathbb{R}^{d}} u(x)\langle\nabla G(x-y), \zeta(x)-\zeta(y)\rangle u(y) \mathrm{d} x \mathrm{~d} y \\
& -2 \iint_{\mathbb{R}^{d} \times \mathbb{R}^{d}} u(x)\langle\nabla G(x-y), \zeta(x)\rangle v(y) \mathrm{d} x \mathrm{~d} y .
\end{aligned}
$$

Exploiting the symmetry $\nabla G(x-y)=-\nabla G(y-x)$ we finally get

$$
\begin{aligned}
\frac{\mathrm{d}}{\mathrm{d} \varepsilon}\left(\frac{1}{2} \int_{\mathbb{R}^{d}}\left|\nabla \psi_{\varepsilon}\right|^{2} \mathrm{~d} x\right)_{\varepsilon=0} & =\iint_{\mathbb{R}^{d} \times \mathbb{R}^{d}} u(x)\langle\nabla G(x-y), \zeta(x)\rangle[u-v](y) \mathrm{d} x \mathrm{~d} y \\
& =\int_{\mathbb{R}^{d}} u\langle\nabla \psi, \zeta\rangle \mathrm{d} x
\end{aligned}
$$

as in our claim, and the proof is complete.

The above restriction at initial data $u^{0}, v^{0} \in L^{2 d /(d+1)}\left(\mathbb{R}^{d}\right)$, which then is inherited by the solutions to later times, is technically essential in order to differentiate under the integral sign with respect to $\varepsilon$-perturbations and retrieve the discrete EulerLagrange equations. Actually this restriction is not purely technical: in (1.1) it seems natural to require the terms $u \nabla \psi, v \nabla \psi$ to be at least in $L^{1}\left(\mathbb{R}^{d}\right)$ at time $t=0$. If $u^{0}, v^{0}$ are both in $L^{p}\left(\mathbb{R}^{d}\right)$ for some $p$ then the integrability for $\nabla \psi$ coming from (HLS-3) is $\nabla \psi \in L^{d p /(d-p)}\left(\mathbb{R}^{d}\right)$, which is optimal since HLS inequalities are. Solving for $p^{\prime}=\frac{d p}{d-p}$ gives exactly the sharp $p=2 d /(d+1)$ exponent. Technically speaking we had to assume initial $L^{r_{0}}\left(\mathbb{R}^{d}\right)$ regularity with slightly better but arbitrarily close $r_{0}>2 d /(d+1)$. This is needed for technical compactness issues, arising later on when we take the limit $h \rightarrow 0$ to retrieve the weak solution $(u, v)=\lim \left(u_{h}, v_{h}\right)$.

In addition to being an approximate solution in the sense of the previous Proposition, the interpolation $\left(u_{h}, v_{h}\right)$ satisfies

Corollary 4.1 (continuous gradient estimate). Fix $m \geq 1$. Then for all $0<h<T$,

$$
\left\|\nabla\left(u_{h}\right)^{m / 2}\right\|_{L^{2}\left(h, T ; L^{2}\left(\mathbb{R}^{d}\right)\right)}+\left\|\nabla\left(v_{h}\right)^{m / 2}\right\|_{L^{2}\left(h, T ; L^{2}\left(\mathbb{R}^{d}\right)\right)} \leq C(T+1)^{\frac{1}{2}}
$$

and

$$
\left\|\nabla\left(u_{h}\right)^{m}\right\|_{L^{2}\left(h, T ; L^{1}\left(\mathbb{R}^{d}\right)\right)}+\left\|\nabla\left(v_{h}\right)^{m}\right\|_{L^{2}\left(h, T ; L^{1}\left(\mathbb{R}^{d}\right)\right)} \leq C(T+1)^{\frac{1}{2}},
$$

for some constant $C=C\left(u^{0}, v^{0}\right)>0$ independent of $h$. 
29 roof. For once the argument requires no distinction between $m>1$ or $m=1$. We only estimate the $u$ component because the computations are identical for $v$.

Since $u_{h}^{m / 2}, \nabla u_{h}^{m / 2} \in L^{2}\left(\mathbb{R}^{d}\right)$ we have

$$
\nabla\left(u_{h}\right)^{m}=2 u_{h}^{\frac{m}{2}} \nabla\left(u_{h}^{\frac{m}{2}}\right) \in L^{1}\left(\mathbb{R}^{d}\right) .
$$

Recalling that $u_{h}^{m / 2}(t)$ is actually bounded in $L^{2}\left(\mathbb{R}^{d}\right)$ uniformly in $t \geq 0$ and $h$, clearly (4.8) will follow from (4.7) and we only establish the latter.

For fixed $0<h<T$ let $N=\lfloor T / h\rfloor$, and recall that the interpolation $z_{h}(t)$ is piecewise constant. Multiplying (3.15) by $h>0$ and summing from $n=0$ to $n=N$ we obtain

$$
\begin{aligned}
\int_{h}^{T} & \left\|\nabla\left(u_{h}\right)^{m / 2}\right\|_{L^{2}\left(\mathbb{R}^{d}\right)}^{2} \mathrm{~d} t \\
& \leq \int_{h}^{(N+1) h}\left\|\nabla\left(u_{h}(t)^{m / 2}\right)\right\|_{L^{2}\left(\mathbb{R}^{d}\right)}^{2} \mathrm{~d} t=\sum_{n=0}^{N-1} h\left\|\nabla\left(u_{h}^{(n+1)}\right)^{m / 2}\right\|_{L^{2}\left(\mathbb{R}^{d}\right)}^{2} \\
& \leq C \sum_{n=0}^{N-1}\left(h+\mathcal{H}\left(u_{h}^{(n)}\right)-\mathcal{H}\left(u_{h}^{(n+1)}\right)+\mathcal{H}\left(v_{h}^{(n)}\right)-\mathcal{H}\left(v_{h}^{(n+1)}\right)\right) \\
& \leq C\left(T+\mathcal{H}\left(u^{0}\right)+\mathcal{H}\left(v^{0}\right)-\mathcal{H}\left(u_{h}^{(N)}\right)-\mathcal{H}\left(v_{h}^{(N)}\right)\right)
\end{aligned}
$$

By Proposition 4.2 the second moments $\mathfrak{m}_{2}\left(u_{h}^{(n)}\right), \mathfrak{m}_{2}\left(v_{h}^{(n)}\right)$ are bounded uniformly in $t, h, n$, hence by the Carleman estimate (3.2) we see that $-\mathcal{H}\left(u_{h}^{(N)}\right)-\mathcal{H}\left(v_{h}^{(N)}\right) \leq C$ in (4.9) and the proof is complete.

We observe that another possible way to retrieve better gradient regularity is to estimate

$$
\left|\int_{\mathbb{R}^{d}} \operatorname{div}(\zeta) u_{h}^{m} \mathrm{~d} x\right|+\left|\int_{\mathbb{R}^{d}} \operatorname{div}(\zeta) v_{h}^{m} \mathrm{~d} x\right| \leq C\|\zeta\|_{L^{p}\left(\mathbb{R}^{d}\right)}
$$

for arbitrary vector-fields $\zeta \in \mathcal{C}_{c}^{\infty}\left(\mathbb{R}^{d} ; \mathbb{R}^{d}\right)$ in the Euler-Lagrange equations (4.5)(4.6), which would estimate by duality $\nabla u_{h}^{m}, \nabla v_{h}^{m} \in L^{p^{\prime}}\left(\mathbb{R}^{d}\right)$, see e.g. [29]. This approach would only improve the previous total variation estimate if $p<\infty$, so that $\left(L^{p^{\prime}}\left(\mathbb{R}^{d}\right)\right)^{\prime}=L^{p}\left(\mathbb{R}^{d}\right)$ and $\mathcal{C}_{c}^{\infty}\left(\mathbb{R}^{d}\right)$ is dense in $L^{p}$. Unfortunately we are here in a limiting situation where essentially $u_{h} \nabla \psi_{h}, v_{h} \nabla \psi_{h} \in L^{1}\left(\mathbb{R}^{d}\right)$ only, so this is not feasible. More precisely, our assumption $u^{0}, v^{0} \in L^{r_{0}}\left(\mathbb{R}^{d}\right)$ with $r_{0}>2 d /(d+1)$ in fact does give slightly better $u_{h} \nabla \psi_{h} \in L^{1+\delta}\left(\mathbb{R}^{d}\right)$ integrability through HLS inequalities (for some $\delta>0$ depending on $r_{0}$ ). But since $r_{0}$ could be arbitrarily close to the critical $2 d /(d+1)$ exponent, $\delta>0$ is arbitrarily small and we shall refrain from taking this technical path.

\section{Convergence to a weak solution}

This section is devoted to the convergence of the previously approximated interpolating solution towards the final weak solution, $(u, v)=\lim _{h \rightarrow 0}\left(u_{h}, v_{h}\right)$ in some suitable topology. Because of the quadratic interaction term and nonlinear diffusion if $m>1$ we will need the following strong convergence

Theorem 4 (Strong convergence). There is a discrete subsequence, still denoted $h \searrow 0$, and functions $u, v$ such that

$$
u_{h}(t, x) \rightarrow u(t, x) \text { and } v_{h}(t, x) \rightarrow v(t, x) \quad \text { a.e. in }(0, \infty) \times \mathbb{R}^{d}
$$


and

$$
\forall 1 \leq p<\infty, 1 \leq q<r_{0}: \quad u_{h}, v_{h} \rightarrow u, v \quad \text { in } L_{l o c}^{p}\left([0, \infty) ; L^{q}\left(\mathbb{R}^{d}\right)\right)
$$

where $r_{0}>\max \{m, 2 d /(d+1)\}$ is the initial integrability as in (2.3).

Observe in particular that $q=m$ and $q=2 d /(d+1)$ are allowed in (5.2), which will be crucial in order to pass to the limit in the Euler-Lagrange equations later on. Roughly speaking, $L^{r}\left(\mathbb{R}^{d}\right)$ integrability suffices to guarantee $L^{q}\left(\mathbb{R}^{d}\right)$ convergence for all $q<r$. Unfortunately $q=\max \{m, 2 d /(d+1)\}$ is a borderline case that we could not treat, and this is why we needed to assume initial $L^{r_{0}}\left(\mathbb{R}^{d}\right)$ integrability for some slightly better but arbitrarily close $r_{0}>\max \{m, 2 d /(d+1)\}$.

Strategy of proof: We will use a compactness criterion in Bochner spaces from [32] that involves

(i) boundedness in $L^{p}(0, T ; X)$ for some strong $X$ topology,

(ii) compactness in $L^{p}(0, T ; Y)$ for a weaker $Y$ space,

(iii) a target intermediate $L^{p}(0, T ; B)$ space with embeddings $X \subset \subset B \subset Y$.

Note that $X \subset \subset B$ will be achieved by space difference quotients and that the key ingredient to obtain boundedness of $\left\{u_{h}, v_{h}\right\}_{h}$ in the strong $X$ topology is the gradient estimate from Corollary 4.1. Compactness in $L^{p}(0, T ; Y)$ will be ensured by an approximated time equi-continuity in some suitable $W^{-s, r^{\prime}}\left(\mathbb{R}^{d}\right)$ space.

We first collect some technical results and then establish Theorem 4 . To begin with, for $m>1$, we set

$$
q_{m}:=1+\frac{1}{m^{\prime}}=1+\frac{m-1}{m} \in(1, m) .
$$

Then there exists $\theta_{m} \in(0,1)$ satisfying

$$
\frac{1}{q_{m}}=\left(1-\theta_{m}\right) \frac{1}{1}+\theta_{m} \frac{1}{m}
$$

and we let

$$
p_{m}:=\frac{2 m}{\theta_{m}}>1
$$

If $\tau_{e}$ denotes the usual shift operator in space

$$
e \in \mathbb{R}^{d}: \quad \tau_{e} w(x):=w(x-e),
$$

we also define the weighted Nikolsk'ii spaces

$$
X_{m}:=\left\{w \in L^{q_{m}}\left(\mathbb{R}^{d}\right): \sup _{e \in \mathbb{R}^{d}}\left\|\tau_{e} w-w\right\|_{L^{q_{m}}}|e|^{-\frac{\theta_{m}}{m}}<\infty, \int_{\mathbb{R}^{d}}|x|^{\frac{2}{m^{\prime}}}|w|^{q_{m}} \mathrm{~d} x<\infty\right\}
$$

endowed with their natural Banach norms with $\theta_{m} / m<1$. By the Riesz-FréchetKolmogorov Theorem we have

$$
X_{m} \subset \subset L^{q_{m}}\left(\mathbb{R}^{d}\right) .
$$

We note that the above choice for $p=p_{m}, q=q_{m}, \theta=\theta_{m}$ is purely technical so we shall go as little as possible into details regarding their explicit values.

For the case $m=1$ one should similarly use

$$
X_{1}:=\left\{w \in L^{1}\left(\mathbb{R}^{d}\right): \nabla w \in L^{1}\left(\mathbb{R}^{d}\right), \int_{\mathbb{R}^{d}}|x|^{2}|w| \mathrm{d} x<\infty\right\} \subset \subset L^{1}\left(\mathbb{R}^{d}\right) .
$$

Since the related argument is fairly easy compared to the nonlinear case, in what follows we will omit the related proof and focus on the nonlinear diffusion case and henceforth we assume $m>1$. Compactness in space will be ensured by 
PProposition 5.1 (Compactness in space). Let $p, q, \theta, X$ as in (5.3)-(5), and fix $T>0$. Then for $h>0$ small enough we have

$$
\left\|u_{h}\right\|_{L^{p}\left(h, T ; X_{m}\right)}+\left\|v_{h}\right\|_{L^{p}\left(h, T ; X_{m}\right)} \leq C_{T}
$$

uniformly in $h$.

Proof. For simplicity we write here $p=p_{m}, q=q_{m}, \theta=\theta_{m}, X=X_{m}$. We first claim that

$$
\left\|u_{h}(t)\right\|_{X} \leq C\left(1+\left\|\nabla u_{h}^{m}(t)\right\|_{L^{1}\left(\mathbb{R}^{d}\right)}^{\theta / m}\right), \text { for } t \geq h .
$$

Indeed since $q \in(1, m)$, it follows immediately from (4.3) that

$$
\left\|u_{h}\right\|_{L^{\infty}\left(0, \infty ; L^{q}\left(\mathbb{R}^{d}\right)\right)} \leq C .
$$

Using (4.3), (4.4), and Hölder inequality we estimate with $q=q_{m}=1+1 / m^{\prime}$

$$
\begin{aligned}
\int_{\mathbb{R}^{d}}|x|^{\frac{2}{m^{\prime}}} u_{h}^{q}(t) \mathrm{d} x & =\int_{\mathbb{R}^{d}}\left(u_{h}(t)|x|^{2}\right)^{\frac{1}{m^{\prime}}} u_{h}(t) \mathrm{d} x \\
& \leq\left(\int_{\mathbb{R}^{d}}|x|^{2} u_{h}(t) \mathrm{d} x\right)^{\frac{1}{m^{\prime}}}\left\|u_{h}(t)\right\|_{L^{m}\left(\mathbb{R}^{d}\right)} \leq C .
\end{aligned}
$$

Fixing $e \in \mathbb{R}^{d}$ and using the convexity inequality $|a-b|^{m} \leq\left.|| a\right|^{m}-|b|^{m} \mid$ for $a, b \geq 0$, we get

$$
\begin{aligned}
\left\|\tau_{e} u_{h}(t)-u_{h}(t)\right\|_{L^{m}\left(\mathbb{R}^{d}\right)} & =\left(\int_{\mathbb{R}^{d}}\left|\tau_{e} u_{h}(t)-u_{h}(t)\right|^{m} \mathrm{~d} x\right)^{\frac{1}{m}} \\
& \leq\left(\int_{\mathbb{R}^{d}}\left|\tau_{e} u_{h}^{m}(t)-u_{h}^{m}(t)\right| \mathrm{d} x\right)^{\frac{1}{m}} \\
& \leq|e|^{1 / m}\left\|\nabla u_{h}^{m}(t)\right\|_{L^{1}\left(\mathbb{R}^{d}\right)^{1 / m}}
\end{aligned}
$$

By (5.4) and $\left\|\tau_{e} u_{h}(t)-u_{h}(t)\right\|_{L^{1}\left(\mathbb{R}^{d}\right)} \leq 2$ we get by interpolation

$$
\begin{aligned}
\left\|\tau_{e} u_{h}(t)-u_{h}(t)\right\|_{L^{q}\left(\mathbb{R}^{d}\right)} & \leq\left\|\tau_{e} u_{h}(t)-u_{h}(t)\right\|_{L^{1}\left(\mathbb{R}^{d}\right)}^{1-\theta} \cdot\left\|\tau_{e} u_{h}(t)-u_{h}(t)\right\|_{L^{m}\left(\mathbb{R}^{d}\right)}^{\theta} \\
& \leq 2|e|^{\frac{\theta}{m}}\left\|\nabla u_{h}^{m}(t)\right\|_{L^{1}\left(\mathbb{R}^{d}\right)}^{\frac{\theta}{m}},
\end{aligned}
$$

thus (5.6) holds as claimed.

Taking now the $L^{p}(h, T)$ norm with $p=p_{m}=2 m / \theta$ in (5.6) and using Corollary 4.1 finally leads to

$$
\left\|u_{h}\right\|_{L^{p}(h, T ; X)} \leq C\left(T+\left\|\nabla u_{h}^{m}\right\|_{L^{2}\left(h, T ; L^{1}\left(\mathbb{R}^{d}\right)\right)}^{2 / p}\right) \leq C_{T} .
$$

The estimate for the $v$ component is again identical.

Next, we turn to compactness in time in a weaker topology. We first have

Proposition 5.2 (Time-equicontinuity in $W^{-s, r^{\prime}}\left(\mathbb{R}^{d}\right)$ ). Let $s, r>0$ be large enough so that

$$
W^{s, r}\left(\mathbb{R}^{d}\right) \subset W^{1,2 m^{\prime}}\left(\mathbb{R}^{d}\right) \cap W^{2, \infty}\left(\mathbb{R}^{d}\right) .
$$

Then

$$
\left\|u_{h}\left(t_{2}\right)-u_{h}\left(t_{1}\right)\right\|_{W^{-s, r^{\prime}}}+\left\|v_{h}\left(t_{2}\right)-v_{h}\left(t_{1}\right)\right\|_{W^{-s, r^{\prime}}} \leq C \sqrt{\left|t_{2}-t_{1}\right|+h}, 0 \leq t_{1} \leq t_{2},
$$

for some $C=C_{s, r}>0$ independent of $t_{1}, t_{2}$ and $h$. 
Proof. The argument is very similar to [10, Lemma 13], beginning with the calculation of the approximate $1 / 2$-Hölder continuity of the sequence $\left\{u_{h}(t)\right\}$. For indices $0<n<n^{\prime}$ let $\nabla q$ denote the optimal map from $u_{h}^{(n)}$ to $u_{h}^{\left(n^{\prime}\right)}$ so that $u_{h}^{\left(n^{\prime}\right)}=(\nabla q)_{\#} u_{h}^{(n)}$. Then

$$
\int_{\mathbb{R}^{d}}\left(u_{h}^{\left(n^{\prime}\right)}-u_{h}^{(n)}\right) \xi \mathrm{d} x=\int_{\mathbb{R}^{d}}(\xi(\nabla q(x))-\xi(x)) u_{h}^{(n)}(x) \mathrm{d} x, \xi \in \mathcal{C}_{c}^{\infty}\left(\mathbb{R}^{d}\right) .
$$

Expanding the integrand on the right,

$$
\begin{aligned}
\xi(x)-\xi(\nabla q(x))=\nabla & \xi(\nabla q(x)) \cdot(x-\nabla q(x)) \\
& +\mathcal{O}\left(|x-\nabla q(x)|^{2}\left\|\nabla^{2} \xi\right\|_{L^{\infty}\left(\mathbb{R}^{d}\right)}\right),
\end{aligned}
$$

Hence

$$
\begin{aligned}
\int_{\mathbb{R}^{d}}\left(u_{h}^{\left(n^{\prime}\right)}-u_{h}^{(n)}\right) \xi \mathrm{d} x= & \int_{\mathbb{R}^{d}}[\xi \circ \nabla q-\xi] u_{h}^{(n)} \mathrm{d} x \\
= & \int_{\mathbb{R}^{d}}\langle\nabla q-\mathrm{Id}, \nabla \xi \circ \nabla q\rangle u_{h}^{(n)} \mathrm{d} x \\
& \quad+\mathcal{O}\left(\left\|\nabla^{2} \xi\right\|_{L^{\infty}\left(\mathbb{R}^{d}\right)}\right) \int_{\mathbb{R}^{d}}|\mathrm{Id}-\nabla q|^{2} u_{h}^{(n)} \mathrm{d} x \\
= & \int_{\mathbb{R}^{d}}\langle\nabla q-\mathrm{Id}, \nabla \xi \circ \nabla q\rangle u_{h}^{(n)} \mathrm{d} x \\
& \quad+\mathcal{O}\left(\left\|\nabla^{2} \xi\right\|_{L^{\infty}\left(\mathbb{R}^{d}\right)} d_{W}\left(u_{h}^{\left(n^{\prime}\right)}, u_{h}^{(n)}\right)^{2}\right) .
\end{aligned}
$$

We further compute by Cauchy-Schwarz and Hölder inequalities

$$
\begin{aligned}
& \left|\int_{\mathbb{R}^{d}}\langle\nabla q-\mathrm{Id}, \nabla \xi \circ \nabla q\rangle u_{h}^{(n)} \mathrm{d} x\right| \\
& \leq\left(\int_{\mathbb{R}^{d}}|\nabla q-\mathrm{Id}|^{2} u_{h}^{(n)} \mathrm{d} x\right)^{\frac{1}{2}} \cdot\left(\int_{\mathbb{R}^{d}}|\nabla \xi \circ \nabla q|^{2} u_{h}^{(n)} \mathrm{d} x\right)^{\frac{1}{2}} \\
& \leq d_{W}\left(u_{h}^{(n)}, u_{h}^{\left(n^{\prime}\right)}\right)\left(\int_{\mathbb{R}^{d}}|\nabla \xi|^{2} u_{h}^{\left(n^{\prime}\right)} \mathrm{d} x\right)^{\frac{1}{2}} \\
& \leq d_{W}\left(u_{h}^{(n)}, u_{h}^{\left(n^{\prime}\right)}\right)\left\|u_{h}^{\left(n^{\prime}\right)}\right\|_{L^{m}\left(\mathbb{R}^{d}\right)}^{\frac{1}{2}}\left\||\nabla \xi|^{2}\right\|_{L^{m^{\prime}}\left(\mathbb{R}^{d}\right)}^{\frac{1}{2}} \\
& \leq C d_{W}\left(u_{h}^{(n)}, u_{h}^{\left(n^{\prime}\right)}\right)\|\nabla \xi\|_{L^{2 m^{\prime}\left(\mathbb{R}^{d}\right)}},
\end{aligned}
$$

Given $0<t_{1}<t_{2}$ and $N_{1}=\left\lfloor t_{1} / h\right\rfloor, N_{2}=\left\lfloor t_{2} / h\right\rfloor$, from (4.1) and the Cauchy Schwarz inequality we get

$$
d_{W}\left(u_{h}^{N_{1}}, u_{h}^{N_{2}}\right) \leq \sum_{n=N_{1}}^{N_{2}-1} d_{W}\left(u_{h}^{(n)}, u_{h}^{(n+1)}\right) \leq C \sqrt{\left|t_{2}-t_{1}\right|+h},
$$

and then

$$
\begin{aligned}
& \left|\int_{\mathbb{R}^{d}}\left(u_{h}\left(t_{2}\right)-u_{h}\left(t_{1}\right)\right) \xi \mathrm{d} x\right| \\
& \quad \leq C\left(\|\nabla \xi\|_{L^{2 m^{\prime}}\left(\mathbb{R}^{d}\right)} \sqrt{\left|t_{2}-t_{1}\right|+h}+\left\|\nabla^{2} \xi\right\|_{L^{\infty}\left(\mathbb{R}^{d}\right)} h\right) .
\end{aligned}
$$


${ }^{2}$ ith our choice $W^{s, r} \subset W^{1,2 m^{\prime}} \cap W^{2, \infty}$ and because $h$ is small we finally obtain

$$
\begin{aligned}
\left|\int_{\mathbb{R}^{d}}\left(u_{h}\left(t_{2}\right)-u_{h}\left(t_{1}\right)\right) \xi \mathrm{d} x\right| & \leq C\left(\sqrt{\left|t_{2}-t_{1}\right|+h}+h\right)\|\xi\|_{W^{s, r}} \\
& \leq C \sqrt{\left|t_{2}-t_{1}\right|+h}\|\xi\|_{W^{s, r}}
\end{aligned}
$$

Our statement follows by density of $\mathcal{C}_{c}^{\infty}\left(\mathbb{R}^{d}\right)$ in $W^{s, r}\left(\mathbb{R}^{d}\right)$ and duality $\left(W^{s, r}\left(\mathbb{R}^{d}\right)\right)^{\prime}=$ $W^{-s, r^{\prime}}\left(\mathbb{R}^{d}\right)$.

We are now in position to prove the desired convergence when $h \rightarrow 0$ :

Proof of Theorem 4 . Once again we only establish the result for the $u$ component. Fix any $0<\delta<T$ and let $q=q_{m}, \theta=\theta_{m}, p=p_{m}, X=X_{m}$ as in (5.3)-(5). Taking $s, r$ large enough such that

$$
W^{s, r}\left(\mathbb{R}^{d}\right) \subset \subset L_{l o c}^{m^{\prime}}\left(\mathbb{R}^{d}\right)
$$

is compact. By truncation, a standard duality argument then ensures that

$$
L^{m}\left(\mathbb{R}^{d}\right) \cap L^{1}\left(\mathbb{R}^{d}\right)\left(\left(1+|x|^{2}\right) \mathrm{d} x\right) \subset \subset W^{-s, r^{\prime}}\left(\mathbb{R}^{d}\right)
$$

is also compact. By Proposition 4.2, we see that there is a fixed $W^{-s, r^{\prime}}\left(\mathbb{R}^{d}\right)$-relatively compact set $K$ such that $u_{h}(t) \in K$ for all $t \geq 0$ and small $h>0$. Therefore, we infer from Proposition 5.2 that a refined version of Arzelà-Ascoli Theorem [2, Proposition 3.3.1] can be applied to conclude that there exists $u \in \mathcal{C}\left([0, T] ; W^{-s, r^{\prime}}\left(\mathbb{R}^{d}\right)\right)$ such that

$$
\forall t \in[0, T], \quad u_{h}(t) \rightarrow u(t) \quad \text { in } \quad W^{-s, r^{\prime}}\left(\mathbb{R}^{d}\right)
$$

for some (discrete) subsequence $h \searrow 0$, not relabeled here for simplicity. This pointwise convergence together with the uniform $L^{m}\left(\mathbb{R}^{d}\right) \cap L^{1}\left(\mathbb{R}^{d},\left(1+|x|^{2}\right) \mathrm{d} x\right)$ bounds and Lebesgue's Dominated Convergence Theorem therefore guarantee strong convergence

$$
u_{h} \rightarrow u \text { in } L^{p}\left(0, T ; W^{-s, r^{\prime}}\left(\mathbb{R}^{d}\right)\right) .
$$

By diagonal extraction we can moreover assume that $u \in \mathcal{C}\left([0, \infty) ; W^{-s, r^{\prime}}\right)$ and that (5.8) holds for all $T>0$.

Choosing $s, r$ large enough we can further assume that

$$
X \subset \subset L^{q}\left(\mathbb{R}^{d}\right) \subset W^{-s, r^{\prime}}\left(\mathbb{R}^{d}\right) .
$$

We recall from Proposition 5.1 that $\left\{u_{h}\right\}_{h}$ is bounded in $L^{p}(\delta, T ; X)$, and by (5.8) it is also relatively compact in $L^{p}\left(\delta, T ; W^{-s, r^{\prime}}\left(\mathbb{R}^{d}\right)\right)$. By [32, Lemma 9] we conclude that $\left\{u_{h}\right\}_{h}$ is relatively compact in the intermediate target space, i-e $u_{h} \rightarrow u$ in $L^{p}\left(\delta, T ; L^{q}\left(\mathbb{R}^{d}\right)\right)$ for some subsequence. By a diagonal extraction we may assume that $u$ is independent of $\delta, T$, and $u_{h} \rightarrow u$ in $L_{l o c}^{p}\left(0, \infty ; L^{q}\left(\mathbb{R}^{d}\right)\right)$. Up to extraction of a further subsequence this classically implies the desired pointwise convergence a.e. $(t, x) \in(0, \infty) \times \mathbb{R}^{d}$.

Let us turn now to the $L_{l o c}^{p}\left([0, \infty) ; L^{q}\left(\mathbb{R}^{d}\right)\right)$ convergence, and fix $1 \leq p<\infty$ and $1 \leq q<r_{0}$ as in our statement (we recall here that $r_{0}>\max \{m, 2 d /(d+1)\}$ is the initial integrability $\left.u^{0}, v^{0} \in L^{r_{0}}\left(\mathbb{R}^{d}\right)\right)$. Once again we only focus on the $u$ component. Thanks to the previous $(t, x)$ a.e. convergence we shall apply Vitali's convergence theorem in fixed bounded intervals $(0, T)$, and we only need to check that the sequence $\left\{u_{h}\right\}$ is tight and uniformly integrable in time and space. Tightness 
in time is obvious in bounded intervals, and tightness in space is easily obtained ${ }^{25}$ Young's inequality

$$
\forall t \geq 0: \quad \int_{\mathbb{R}^{d}} u_{h}^{q}|x|^{2 \varepsilon} \mathrm{d} x=\int_{\mathbb{R}^{d}} \underbrace{u_{h}^{q-\varepsilon}}_{\in L^{(1 / \varepsilon)^{\prime}}} \underbrace{\left(u_{h}|x|^{2}\right)^{\varepsilon}}_{\in L^{1 / \varepsilon}} \mathrm{d} x \leq C
$$

uniformly in $h, t$ for some suitably small $\varepsilon>0$. Here we used the uniform bounds on the second moment $\mathfrak{m}_{2}\left(u_{h}\right) \leq C$ and $q-\varepsilon \leq q<r_{0}$ to control $\left\|u_{h}(t)^{q-\varepsilon}\right\|_{L^{(1 / \varepsilon)^{\prime}}\left(\mathbb{R}^{d}\right)}$ by local uniform bounds $\left\|u_{h}(t)\right\|_{L^{r_{0}\left(\mathbb{R}^{d}\right)}} \leq C_{T}$ (obtained by propagation of initial integrability, Proposition 4.3). The same propagation of integrability gives uniform bounds $\left\|u_{h}\right\|_{L^{\infty}(0, T) ; L^{r_{0}\left(\mathbb{R}^{d}\right)}} \leq C$, thus by immediate $L^{1} L^{r_{0}}$ interpolation we obtain equi-integrability in the form

$$
\left\|u_{h}\right\|_{L^{p+\varepsilon}\left(0, T ; L^{q+\varepsilon}\left(\mathbb{R}^{d}\right)\right)} \leq C_{T}
$$

for $\varepsilon>0$ suitably small (essentially such that $1 \leq q+\varepsilon<r_{0}$ ). Applying Vitali's convergence theorem gives strong convergence $u_{h} \rightarrow u$ in $L^{p}\left([0, T) ; L^{q}\left(\mathbb{R}^{d}\right)\right)$ as desired and the proof is complete.

We can now prove our main result. The proof of Theorem 3 is identical to that of Theorem 2 so we only establish the latter.

Proof of Theorem Q Step 1: convergence. Recall that $u_{h}(t), v_{h}(t) \in \mathcal{K}$ for all $t, h$, and that $\mathcal{K}$ is $L^{1}$-weak relatively compact. Using the approximate $1 / 2$ Hölder equicontinuity (5.7) and applying the previous refined Arzelà-Ascoli theorem, we can extract a subsequence such that

$$
\forall t \geq 0: \quad u_{h}(t), v_{h}(t) \rightarrow u(t), v(t) \text { in } L^{1}\left(\mathbb{R}^{d}\right)
$$

for some $u, v \in \mathcal{C}\left(0, T ; \mathcal{P}\left(\mathbb{R}^{d}\right)\right)$ and $u(t), v(t) \in \mathcal{K}$ for all times. This entails the $L^{\infty}\left(0, T ; L^{m}\left(\mathbb{R}^{d}\right) \cap L^{1}\left(\mathbb{R}^{d}\right)\left(\left(1+|x|^{2}\right) \mathrm{d} x\right)\right)$ bounds. By standard truncation arguments we also get that $u(t), v(t)$ are probability measures for all times, and because $d_{W}^{2}$ is l.s.c for the $L^{1}$ - weak convergence we can moreover take the limit in (4.2) to deduce that $t \mapsto u(t), v(t)$ are $1 / 2$-Hölder continuous in $\left(\mathcal{P}, d_{W}\right)$. Since $u_{h}(0), v_{h}(0)=u^{0}, v^{0}$ we can take the limit $u(0), v(0)=u^{0}, v^{0}$, which together with $u, v \in \mathcal{C}^{1 / 2}([0, \infty) ; \mathcal{P})$ shows that the limit $u, v$ satisfies the initial condition at least in the sense of measures as desired.

We claim now that

$$
\nabla u_{h}^{m}, \nabla v_{h}^{m} \rightarrow \nabla u^{m}, \nabla v^{m} \quad \text { in } L^{2}\left(\delta, T ; L^{1}\left(\mathbb{R}^{d}\right)\right)
$$

for all $0<\delta<T$, and also

$$
\nabla u^{m}, \nabla v^{m} \in L^{2}\left(0, T ; L^{1}\left(\mathbb{R}^{d}\right)\right) .
$$

To see this fix a test function $\varphi \in L^{2}\left(\delta, T ; L^{\infty}\left(\mathbb{R}^{d}\right)\right)$, and write for small $h>0$

$$
\int_{\delta}^{T} \int_{\mathbb{R}^{d}}\left(\nabla u_{h}^{m}\right) \varphi \mathrm{d} x \mathrm{~d} t=\int_{\delta}^{T} \int_{\mathbb{R}^{d}} 2 \nabla u_{h}^{m / 2}\left(u_{h}^{m / 2} \varphi\right) \mathrm{d} x \mathrm{~d} t
$$

By Corollary 4.1 and pointwise a.e convergence $u_{h} \rightarrow u$ we can assume $\nabla u_{h}^{m / 2} \rightarrow$ $\nabla u^{m / 2}$ in $L^{2}\left(\delta, T ; L^{2}\left(\mathbb{R}^{d}\right)\right)$ for fixed $0<\delta<T$, and by diagonal extraction we can assume that the limit $\nabla u^{m / 2}$ is independent of $\delta, T$. By (5.2) with $q=m<r_{0}$ it is 
${ }_{\text {easy }}^{26}$ to get $u_{h}^{m / 2} \varphi \rightarrow u^{m / 2} \varphi$ in $L^{2}\left(\delta, T ; L^{2}\left(\mathbb{R}^{d}\right)\right)$. As a consequence we can pass to the limit

$$
\int_{\delta}^{T} \int_{\mathbb{R}^{d}}\left(\nabla u_{h}^{m}\right) \varphi \mathrm{d} x \mathrm{~d} t \underset{h \searrow 0}{\rightarrow} 2 \int_{\delta}^{T} \int_{\mathbb{R}^{d}} \nabla u^{m / 2}\left(u^{m / 2} \varphi\right) \mathrm{d} x \mathrm{~d} t=\int_{\delta}^{T} \int_{\mathbb{R}^{d}} \nabla u^{m} \varphi \mathrm{d} x \mathrm{~d} t
$$

to obtain (5.9). In particular by Corollary 4.1] we see that $\forall 0<\delta<T$, it holds

$$
\begin{aligned}
\left\|\nabla u^{m}\right\|_{L^{2}\left(\delta, T ; L^{1}\left(\mathbb{R}^{d}\right)\right)} & \leq \liminf _{h \searrow 0}\left\|\nabla u_{h}^{m}\right\|_{L^{2}\left(\delta, T ; L^{1}\left(\mathbb{R}^{d}\right)\right)} \\
& \leq 2 \liminf _{h \searrow 0}\left\|u_{h}^{m / 2}\right\|_{L^{\infty}\left(\delta, T ; L^{2}\left(\mathbb{R}^{d}\right)\right)}\left\|\nabla u_{h}^{m / 2}\right\|_{L^{2}\left(\delta, T ; L^{2}\left(\mathbb{R}^{d}\right)\right)} \\
& \leq C(1+T)^{1 / 2}
\end{aligned}
$$

uniformly in $\delta>0$, whence $\nabla u^{m} \in L^{2}\left(0, T ; L^{1}\left(\mathbb{R}^{d}\right)\right)$ for all $T>0$.

For the drift terms, recall from (2.1) that $\nabla U(x), \nabla V(x)$ are at least locally bounded. From Theorem 4 we have $u_{h}, v_{h} \rightarrow u, v$ at least in $L^{1}\left(0, T ; L^{1}\left(\mathbb{R}^{d}\right)\right)$, thus $u_{h} \nabla U, v_{h} \nabla V \rightarrow u \nabla U, v \nabla V$ in $L^{1}\left(0, \infty ; L_{l o c}^{1}\left(\mathbb{R}^{d}\right)\right)$ when tested with compactly supported functions $\varphi(x)$. Moreover by uniform bounds on the second moments and linear behavior of $\nabla U, \nabla V$ it is easy to check that the limit $u \nabla U, v \nabla V \in$ $L^{\infty}\left(0, \infty ; L^{1}\left(\mathbb{R}^{d}\right)\right)$.

Regarding now the coupling terms $u_{h} \nabla \psi_{h}, v_{h} \nabla \psi_{h}$, note from (5.2) with $q=$ $2 d /(d+1)<r_{0}$ that we have in particular $u_{h}, v_{h} \rightarrow u, v$ in $L^{p}\left(\delta, T ; L^{2 d / d+1}\left(\mathbb{R}^{d}\right)\right)$ for all $p \in[1, \infty)$. By strong $L^{2 d /(d+1)}\left(\mathbb{R}^{d}\right) \rightarrow L^{2 d /(d-1)}\left(\mathbb{R}^{d}\right)$ continuity in (HLS-3) we thus obtain $\nabla \psi_{h}=(\nabla G) *\left[u_{h}-v_{h}\right] \rightarrow(\nabla G) *[u-v]=\nabla \psi$ in $L^{p}\left(\delta, T ; L^{2 d /(d-1)}\left(\mathbb{R}^{d}\right)\right)$ for all $p \in[1, \infty)$, so by Hölder inequality

$$
u_{h} \nabla \psi_{h}, v_{h} \nabla \psi_{h} \rightarrow u \nabla \psi, v \nabla \psi \quad \text { in } L^{p}\left(\delta, T ; L^{1}\left(\mathbb{R}^{d}\right)\right)
$$

for all $p \in[1, \infty)$ and $0<\delta<T$. Using the $L^{\infty}\left(\delta, T ; L^{2 d /(d+1)}\left(\mathbb{R}^{d}\right)\right)$ bounds for $u_{h}, v_{h}$ this gives $L^{p}\left(\delta, T ; L^{1}\left(\mathbb{R}^{d}\right)\right)$ bounds uniformly in $p \geq 1$ and $\delta>0$, thus $u \nabla \psi, v \nabla \psi \in$ $L^{\infty}\left(0, T ; L^{1}\left(\mathbb{R}^{d}\right)\right) \subset L^{2}\left(0, T ; L^{1}\left(\mathbb{R}^{d}\right)\right)$ for all $T>0$.

Step 2: the weak solution. Fix any test-function $\varphi \in \mathcal{C}_{c}^{\infty}\left(\mathbb{R}^{d}\right)$ and $0<T_{1}<T_{2}$, and let $N_{1}=\left\lfloor T_{1} / h\right\rfloor, N_{2}=\left\lfloor T_{2} / h\right\rfloor$. Let $\nabla q^{(n)}$ be the optimal map in $u_{h}^{(n+1)}=$ $\left(\nabla q^{(n)}\right)_{\#} u_{h}^{(n)}$. Expanding

$$
\begin{aligned}
\varphi(x)-\varphi\left(\nabla q^{(n)}(x)\right)=( & \left.\nabla \varphi\left(\nabla q^{(n)}(x)\right)\right) \cdot\left(x-\nabla q^{(n)}(x)\right) \\
& +\mathcal{O}\left(\left|x-\nabla q^{(n)}(x)\right|^{2}\left\|D^{2} \varphi\right\|_{L^{\infty}\left(\mathbb{R}^{d}\right)}\right) .
\end{aligned}
$$


Taking $\zeta=\nabla \varphi$ in the Euler-Lagrange equation (4.5), and summing from $n=N_{1}$ to $n=N_{2}-1$ we compute by (4.1), (4.5) and (5.10) that

$$
\begin{aligned}
& \int_{\mathbb{R}^{d}}\left(u_{h}\left(T_{2}\right)-u_{h}\left(T_{1}\right)\right) \varphi \mathrm{d} x \\
&= \int_{\mathbb{R}^{d}}\left(u_{h}^{\left(N_{2}\right)}-u_{h}^{\left(N_{1}\right)}\right) \varphi \mathrm{d} x \\
&= \sum_{n=N_{1}}^{N_{2}-1} \int_{\mathbb{R}^{d}}\left(u_{h}^{(n+1)}-u_{h}^{(n)}\right) \varphi \mathrm{d} x \\
&= \sum_{n=N_{1}}^{N_{2}-1} \int_{\mathbb{R}^{d}}\left[\varphi \circ \nabla q^{(n)}-\varphi\right] u_{h}^{(n)} \mathrm{d} x \\
&= \sum_{n=N_{1}}^{N_{2}-1} \int_{\mathbb{R}^{d}}\left\langle\nabla q^{(n)}-\mathrm{Id}, \nabla \varphi \circ \nabla q^{(n)}\right\rangle u_{h}^{(n)} \mathrm{d} x \\
&+\sum_{n=N_{1}}^{N_{2}-1} \mathcal{O}\left(\left\|D^{2} \varphi\right\|_{L^{\infty}\left(\mathbb{R}^{d}\right)} d_{W}^{2}\left(u_{h}^{(n)}, u_{h}^{(n+1)}\right)\right) \\
&= \sum_{n=N_{1}}^{N_{2}-1} h \int_{\mathbb{R}^{d}}\left[\Delta \varphi\left(u_{h}^{(n+1)}\right)^{m}-u_{h}^{(n+1)}\langle\nabla U, \nabla \varphi\rangle-u_{h}^{(n+1)}\left\langle\nabla \psi_{h}^{(n+1)}, \nabla \varphi\right\rangle\right] \mathrm{d} x \\
& \quad+\mathcal{O}\left(h\left\|D^{2} \varphi\right\|_{L^{\infty}\left(\mathbb{R}^{d}\right)}\right) .
\end{aligned}
$$

Integrating by parts and exploiting (4.1), at the continuous level this becomes

$$
\begin{aligned}
& \mathcal{O}\left(h\left\|D^{2} \varphi\right\|_{L^{\infty}\left(\mathbb{R}^{d}\right)}\right)+\int_{\mathbb{R}^{d}}\left(u_{h}\left(T_{2}\right)-u_{h}\left(T_{1}\right)\right) \varphi \mathrm{d} x \\
& =-\int_{N_{1} h}^{N_{2} h} \int_{\mathbb{R}^{d}}\left[\left\langle\nabla\left(u_{h}(t)\right)^{m}, \nabla \varphi\right\rangle+\langle\nabla U, \nabla \varphi\rangle u_{h}(t)+u_{h}(t)\left\langle\nabla \psi_{h}(t), \nabla \varphi\right\rangle\right] \mathrm{d} x \mathrm{~d} t .
\end{aligned}
$$

By step 1 we can take $h \rightarrow 0$ as

$$
\begin{aligned}
\int_{\mathbb{R}^{d}}\left(u\left(T_{2}\right)-u\left(T_{1}\right)\right) \varphi \mathrm{d} x \\
=-\int_{T_{1}}^{T_{2}} \int_{\mathbb{R}^{d}}\left(\left\langle\nabla u^{m}, \nabla \varphi\right\rangle+u\langle\nabla U, \nabla \varphi\rangle+u\langle\nabla \psi, \nabla \varphi\rangle\right) \mathrm{d} x \mathrm{~d} t
\end{aligned}
$$

to obtain (2.8). The equation for $v$ is similarly obtained.

Step 3: Energy bounds and further regularity. We first establish the energy bound (2.11). Arguing as in step 1, using (5.2) with $q=2 d /(d+2)<r_{0}$, and (HLS-3) it is easy to conclude that $\nabla \psi_{h} \rightarrow \nabla \psi$ in $L_{l o c}^{p}\left(0, \infty ; L^{2}\left(\mathbb{R}^{d}\right)\right)$ for all $p \in[1, \infty)$. In particular $\nabla \Psi_{h}(t) \rightarrow \nabla \Psi(t)$ in $L^{2}\left(\mathbb{R}^{d}\right)$ and

$$
\mathcal{E}_{\mathrm{cpl}}\left(u_{h}(t), v_{h}(t)\right) \rightarrow \mathcal{E}_{\mathrm{cpl}}(u(t), v(t)) \quad \text { a.e. } t \geq 0
$$

From the energy control $\left\|\nabla \psi_{h}\right\|_{L^{\infty}\left(0, \infty ; L^{2}\left(\mathbb{R}^{d}\right)\right)} \leq C$ we also have $\nabla \psi \in L^{\infty}\left(0, \infty ; L^{2}\left(\mathbb{R}^{d}\right)\right)$. Similarly, from the $L_{l o c}^{p}\left(0, \infty ; L^{q}\left(\mathbb{R}^{d}\right)\right)$ with $q=m<r_{0}$ it is easy to get

$$
\mathcal{E}_{\text {diff }}\left(u_{h}(t), v_{h}(t)\right) \rightarrow \mathcal{E}_{\text {diff }}(u(t), v(t)) \quad \text { a.e. } t \geq 0 .
$$


For the potential energy, we have $0 \leq u_{h}(t, x) U(x) \rightarrow u(t, x) U(x)$ a.e. $x \in \mathbb{R}^{d}$ for a.e. fixed $t \geq 0$, and similarly for $v_{h} V$. By Fatou's lemma we conclude that

$$
\mathcal{E}_{\text {ext }}(u(t), v(t)) \leq \liminf _{h \rightarrow 0} \mathcal{E}_{\text {ext }}\left(u_{h}(t), v_{h}(t)\right) \quad \text { a.e. } t \geq 0 .
$$

Summing $\mathcal{E}=\mathcal{E}_{\text {diff }}+\mathcal{E}_{\text {ext }}+\mathcal{E}_{\text {cpl }}$ with $\mathcal{E}\left(u_{h}(t), v_{h}(t)\right) \leq \mathcal{E}\left(u^{0}, v^{0}\right)$ at the discrete level finally entails the desired energy bound (2.11). Note that the potential energy $\mathcal{E}_{\text {ext }}$ is the most problematic term, because we cannot a priori conclude equality $\mathcal{E}_{\text {ext }}(u(t), v(t))=\lim \mathcal{E}_{\text {ext }}\left(u_{h}(t), v_{h}(t)\right)$ in the last display (in fact strong convergence would imply convergence of the second moments, thus convergence in $d_{W}$ ). As a consequence we only retrieve the one-sided inequality, and we are unable to conclude that the total energy is monotone nonincreasing in the limit.

Turning now to the propagation of initial regularity (2.12), assume that the initial datum $u^{0}, v^{0} \in L^{p}\left(\mathbb{R}^{d}\right)$ for some $p \in[1, \infty]$. If $p<\infty$ then Proposition 4.3 bounds $u_{h}(t), v_{h}(t)$ in $L^{p}\left(\mathbb{R}^{d}\right)$ uniformly in $h$ with exponential control

$$
\sup _{t \in[0, \tau]}\left(\left\|u_{h}(t)\right\|_{L^{p}\left(\mathbb{R}^{d}\right)}+\left\|v_{h}(t)\right\|_{L^{p}\left(\mathbb{R}^{d}\right)}\right) \leq C e^{\lambda \tau}\left(\left\|u^{0}\right\|_{L^{p}\left(\mathbb{R}^{d}\right)}+\left\|v^{0}\right\|_{L^{p}\left(\mathbb{R}^{d}\right)}\right) .
$$

Up to extraction of a further subsequence we can assume that $u_{h}, v_{h} \stackrel{*}{\rightarrow} u, v$ in $L_{l o c}^{\infty}\left([0, \infty) ; L^{p}\left(\mathbb{R}^{d}\right)\right)$, which immediately gives (2.12). If now $u^{0}, v^{0} \in L^{\infty}\left(\mathbb{R}^{d}\right)$ clearly (2.12) holds for arbitrarily large $p$. Our claim then easily follows by letting $p \rightarrow \infty$ and the proof is achieved.

\section{Appendix}

For $p>1$ let $L_{w}^{p}\left(\mathbb{R}^{d}\right)$ be the weak- $L^{p}$ spaces, which coincide with the usual Lorentz space $L^{p, \infty}\left(\mathbb{R}^{d}\right)$. The natural Banach norm is

$$
\|w\|_{L_{w}^{p}\left(\mathbb{R}^{d}\right)}=\|w\|_{L^{p, \infty}\left(\mathbb{R}^{d}\right)}=\sup _{t>0}\left\{t^{1 / p} w^{*}(t)\right\}
$$

where $w^{*}(t)$ is the symmetric-decreasing rearrangement of $w(x)$.

Proposition 6.1. Denoting $\Phi=(-\Delta)^{-1} w=G * w$, the Dirichlet energy

$$
w \in L^{1}\left(\mathbb{R}^{d}\right) \mapsto \mathcal{E}_{D}(w)=\int_{\mathbb{R}^{d}}|\nabla \Phi|^{2} \mathrm{~d} x \in[0,+\infty]
$$

is lower semi-continuous for weak $L^{1}$ convergence.

Proof. Let $w_{n} \rightarrow w$ in $L^{1}\left(\mathbb{R}^{d}\right)$. If $\liminf \mathcal{E}_{D}\left(w_{n}\right)=+\infty$ our statement is trivial, so up to extraction of a subsequence we may assume that $\liminf \mathcal{E}_{D}\left(w_{n}\right)=\lim \mathcal{E}_{D}\left(w_{n}\right)=$ $C<+\infty$, in particular we have that

$$
\lim \left\|\nabla \Phi_{n}\right\|_{L^{2}\left(\mathbb{R}^{d}\right)}^{2}=\liminf \mathcal{E}_{D}\left(w_{n}\right)<+\infty .
$$

Now since $w_{n} \rightarrow w$ in $L^{1}\left(\mathbb{R}^{d}\right)$ we see that $w_{n}$ is bounded in $L^{1}\left(\mathbb{R}^{d}\right)$, hence by (HLS-2),

$$
\left\|\Phi_{n}\right\|_{L_{w}^{d /(d-2)}\left(\mathbb{R}^{d}\right)} \leq C\left\|w_{n}\right\|_{L^{1}\left(\mathbb{R}^{d}\right)} \leq C .
$$

Since $L_{w}^{d /(d-2)}\left(\mathbb{R}^{d}\right)=L^{d /(d-2), \infty}\left(\mathbb{R}^{d}\right)=\left(L^{d / 2,1}\left(\mathbb{R}^{d}\right)\right)^{\prime}$ is a topological dual we can also assume, by the Banach-Alaoglu theorem, and up to a further subsequence, that

$$
\Phi_{n} \stackrel{*}{\rightarrow} \Phi \quad \text { in } L_{w}^{d /(d-2)}\left(\mathbb{R}^{d}\right) .
$$

By (6.2) and up to a subsequence we see that

$$
\|\nabla \Phi\|_{L^{2}\left(\mathbb{R}^{d}\right)}^{2} \leq \liminf \left\|\nabla \Phi_{n}\right\|_{L^{2}\left(\mathbb{R}^{d}\right)}^{2}=\liminf \mathcal{E}_{D}\left(w_{n}\right) .
$$


As a consequence it suffices to prove that $\Phi=G * w$, since then $\mathcal{E}_{D}(w)=\|\nabla \Phi\|_{L^{2}\left(\mathbb{R}^{d}\right)}^{2} \leq$ $\lim \inf \mathcal{E}_{D}\left(w_{n}\right)$.

Set $\tilde{\Phi}=G * w \in L_{w}^{d /(d-2)}\left(\mathbb{R}^{d}\right)$ and let us prove that $\Phi-\tilde{\Phi}=0$. Since $-\Delta \Phi_{n}=$ $w_{n} \rightarrow w=-\Delta \tilde{\Phi}$ in $L^{1}\left(\mathbb{R}^{d}\right)$ we have in particular that $\Phi-\tilde{\Phi}$ is harmonic. Because harmonic tempered distributions are polynomials and $L_{w}^{d /(d-2)}\left(\mathbb{R}^{d}\right) \subset \mathcal{S}^{\prime}\left(\mathbb{R}^{d}\right)$ we get that $\Phi-\tilde{\Phi} \in L_{w}^{d /(d-2)}\left(\mathbb{R}^{d}\right)$ is polynomial. By (6.1) we see that the polynomial $\Phi-\tilde{\Phi}$ decays at infinity, hence $\Phi-\tilde{\Phi}=0$ as claimed and the proof is complete.

Acknowledgments. We would like to thank Adrien Blanchet for fruitful discussions and also his hospitality to LM. We are very grateful to the careful reading and important suggestions from the anonymous referee. We thank Marcus Wunsch for introducing us to this problem. This work is partially supported by DMS 0806703, DMS 0635983, DMS 091501, DMS-1217066, DMS 1419053, OISE 0967140, Portugal/CMU program, and FCT SFRH/BPD/88207/2012.

\section{REFERENCES}

[1] Luigi Ambrosio and Nicola Gigli. A user's guide to optimal transport. In Modelling and optimisation of flows on networks, volume 2062 of Lecture Notes in Math., pages 1-155. Springer, Heidelberg, 2013.

[2] Luigi Ambrosio, Nicola Gigli, and Giuseppe Savaré. Gradient flows in metric spaces and in the space of probability measures. Lectures in Mathematics ETH Zürich. Birkhäuser Verlag, Basel, second edition, 2008.

[3] Anton Arnold, Peter Markowich, and Giuseppe Toscani. On large time asymptotics for driftdiffusion-Poisson systems. In Proceedings of the Fifth International Workshop on Mathematical Aspects of Fluid and Plasma Dynamics (Maui, HI, 1998), volume 29, pages 571-581, 2000.

[4] Anton Arnold, Peter Markowich, Giuseppe Toscani, and Andreas Unterreiter. On convex Sobolev inequalities and the rate of convergence to equilibrium for Fokker-Planck type equations. Comm. Partial Differential Equations, 26(1-2):43-100, 2001.

[5] Jean-David Benamou and Yann Brenier. A computational fluid mechanics solution to the Monge-Kantorovich mass transfer problem. Numer. Math., 84(3):375-393, 2000.

[6] Piotr Biler and Jean Dolbeault. Long time behavior of solutions of Nernst-Planck and DebyeHückel drift-diffusion systems. Ann. Henri Poincaré, (3):461-472, 2000.

[7] Piotr Biler, Jean Dolbeault, and Peter A. Markowich. Large time asymptotics of nonlinear drift-diffusion systems with Poisson coupling. Transport Theory Statist. Phys., 30(4-6):521536, 2001. The Sixteenth International Conference on Transport Theory, Part II (Atlanta, GA, 1999).

[8] Adrien Blanchet, Vincent Calvez, and José A. Carrillo. Convergence of the mass-transport steepest descent scheme for the subcritical Patlak-Keller-Segel model. SIAM J. Numer. Anal., 46(2):691-721, 2008.

[9] Adrien Blanchet, José Antonio Carrillo, David Kinderlehrer, Michal Kowalczyk, Philippe Laurençot, and Stefano Lisini. A hybrid variational principle for the keller-segel system in $\mathbb{R}^{2}$. To appear, arXiv:140\%.5562 [math.AP], 2014.

[10] Adrien Blanchet and Philippe Laurençot. The parabolic-parabolic Keller-Segel system with critical diffusion as a gradient flow in $\mathbb{R}^{d}, d \geq 3$. Comm. Partial Differential Equations, 38(4):658-686, 2013.

[11] José A. Carrillo, Robert J. McCann, and Cédric Villani. Kinetic equilibration rates for granular media and related equations: entropy dissipation and mass transportation estimates. Rev. Mat. Iberoamericana, 19(3):971-1018, 2003.

[12] Marco Di Francesco and Marcus Wunsch. Large time behavior in Wasserstein spaces and relative entropy for bipolar drift-diffusion-Poisson models. Monatsh. Math., 154(1):39-50, 2008.

[13] Weifu Fang and Kazufumi Ito. On the time-dependent drift-diffusion model for semiconductors. J. Differential Equations, 117(2):245-280, 1995.

[14] Herbert Gajewski. On the uniqueness of solutions to the drift-diffusion model of semiconductor devices. Math. Models Methods Appl. Sci., 4(1):121-133, 1994. 
[15] Stuart Hastings, David Kinderlehrer, and J. Bryce McLeod. Diffusion mediated transport in multiple state systems. SIAM J. Math. Anal., 39(4):1208-1230, 2007/08.

[16] Richard Jordan, David Kinderlehrer, and Felix Otto. The variational formulation of the FokkerPlanck equation. SIAM J. Math. Anal., 29(1):1-17, 1998.

[17] Ansgar Jüngel. A nonlinear drift-diffusion system with electric convection arising in electrophoretic and semiconductor modeling. Math. Nachr., 185:85-110, 1997.

[18] Ansgar Jüngel. Quasi-hydrodynamic semiconductor equations. Progress in Nonlinear Differential Equations and their Applications, 41. Birkhäuser Verlag, Basel, 2001.

[19] Carlos E. Kenig. Harmonic analysis techniques for second order elliptic boundary value problems, volume 83 of CBMS Regional Conference Series in Mathematics. Published for the Conference Board of the Mathematical Sciences, Washington, DC; by the American Mathematical Society, Providence, RI, 1994.

[20] David Kinderlehrer and Michal Kowalczyk. The Janossy effect and hybrid variational principles. Discrete Contin. Dyn. Syst. Ser. B, 11(1):153-176, JAN 2009.

[21] Masaki Kurokiba and Takayoshi Ogawa. Well-posedness for the drift-diffusion system in $L^{p}$ arising from the semiconductor device simulation. J. Math. Anal. Appl., 342(2):1052-1067, 2008.

[22] Philippe Laurençot and Bogdan-Vasile Matioc. A gradient flow approach to a thin film approximation of the Muskat problem. Calc. Var. Partial Differential Equations, 47(1-2):319-341, 2013.

[23] Elliott H. Lieb and Michael Loss. Analysis, volume 14 of Graduate Studies in Mathematics. American Mathematical Society, Providence, RI, second edition, 2001.

[24] P. A. Markowich, C. A. Ringhofer, and C. Schmeiser. Semiconductor equations. SpringerVerlag, Vienna, 1990.

[25] Daniel Matthes, Robert J. McCann, and Giuseppe Savaré. A family of nonlinear fourth order equations of gradient flow type. Comm. Partial Differential Equations, 34(10-12):1352-1397, 2009.

[26] Daniel Matthes and Jonathan Zinsl. Exponential convergence to equilibrium in a coupled gradient flow system modelling chemotaxis. To appear, arXiv:1310.3977 [math.AP], 2014.

[27] Robert J. McCann. A convexity principle for interacting gases. Adv. Math., 128(1):153-179, 1997.

[28] Alexander Mielke. A gradient structure for reaction-diffusion systems and for energy-driftdiffusion systems. Nonlinearity, 24(4):1329-1346, 2011.

[29] Felix Otto. Dynamics of labyrinthine pattern formation in magnetic fluids: a mean-field theory. Arch. Rational Mech. Anal., 141(1):63-103, 1998.

[30] Felix Otto. The geometry of dissipative evolution equations: the porous medium equation. Comm. Partial Differential Equations, 26(1-2):101-174, 2001.

[31] M. Schmuck. New porous medium Poisson-Nernst-Planck equations for strongly oscillating electric potentials. J. Math. Phys., 54(2):021504, 21, 2013.

[32] Jacques Simon. Compact sets in the space $L^{p}(0, T ; B)$. Ann. Mat. Pura Appl. (4), 146:65-96, 1987.

[33] Elias M. Stein. Singular integrals and differentiability properties of functions. Princeton Mathematical Series, No. 30. Princeton University Press, Princeton, N.J., 1970.

[34] Juan Luis Vázquez. The porous medium equation. Oxford Mathematical Monographs. The Clarendon Press, Oxford University Press, Oxford, 2007. Mathematical theory.

[35] Cédric Villani. Topics in optimal transportation, volume 58 of Graduate Studies in Mathematics. American Mathematical Society, Providence, RI, 2003.

[36] Shixin Xu, Ping Sheng, and Chun Liu. An energetic variational approach for ion transport. Commun. Math. Sci., 12(4):779-789, 2014.

[37] Jonathan Zinsl. Existence of solutions for a nonlinear system of parabolic equations with gradient flow structure. Monatshefte für Mathematik, 174(4):653-679, 2014. 
Department of Mathematical Sciences, Carnegie Mellon University, Pittsburghi, PA 15213, USA

CAMgsd Instituto Superior Técnico, Av. Rovisco Pais 1049-001 Lisbon, Portugal

Department of Mathematics and Statistics, Old Dominion University, 2300 EngiNeering \& Computational Sciences Bldg Norfolk, VA 23529, USA

E-mail address: davidk@andrew.cmu.edu

E-mail address: leonard.monsaingeon@ist.utl.pt

E-mail address: x2xu@odu.edu 